\title{
Evaluation and Development of Non-Reflective Boundary Conditions for Aeroacoustic Simulations
}

\author{
James Gill, ${ }^{*}$ Ryu Fattah, ${ }^{\dagger}$ and Xin Zhang ${ }^{\ddagger}$ \\ Faculty of Engineering and the Environment, \\ University of Southampton, Hampshire, SO16 7QF, UK.
}

\begin{abstract}
The performance of several non-reflective acoustic boundary conditions is quantitatively compared using a plane wave test configuration. The study uses a high-order linearized Euler equation solver to find a non-reflective boundary condition which gives good performance in a variety of cases. The performance for acoustic waves with varying frequency and incident angle is compared in flows with varying Mach number. The performance of buffer zone, far-field, and characteristic non-reflective methods is compared. Some non-reflective boundary methods, such as buffer zones, contain tuneable parameters that are optimized in the current work. This provides a more comprehensive evaluation than previous studies which used constant values of the tuneable parameters. A new generic non-reflective zonal characteristic boundary condition is proposed and is shown to give improved performance in comparison to other tested methods. The performance of the proposed boundary condition is also demonstrated in a two-dimensional airfoil turbulence-interaction case that includes vortical waves leaving the domain, and in a three-dimensional duct mode case.
\end{abstract}

Keywords: computational aeroacoustics, non-reflective boundary conditions, linearized Euler equations

\section{Introduction}

$\mathrm{T}$ HERE is a requirement in computational aeroacoustic (CAA) simulations for effective non-reflecting boundary conditions at the edges of a domain. A computational domain is usually truncated (i.e. it does not stretch to infinity) to reduce the computational cost. This truncation, combined with the use of numerical discretization schemes that are optimized for minimal dispersion and dissipation errors, makes simulations sensitive to spurious reflections from outgoing waves impacting the domain boundaries. CAA investigations have defined a variety of non-reflective boundary conditions in order to prevent contamination of numerical solutions from spurious reflections of acoustic, vortical, or entropy waves.

Different methods have previously been used in order to obtain a non-reflective boundary condition. These methods have been developed for various governing equations, including the Navier-Stokes, Euler, and linearized Euler equations (LEE). In this paper we concentrate on applications to the LEE. Despite the various methods that have been developed, no method currently exists to remove all reflections for all cases. Factors such as the frequency, meanflow non-uniformity, wave angle, and Mach number, can affect the performance. ${ }^{1}$ This work aims to provide a thorough quantitative comparison of the performance of different non-reflective techniques. The following are addressed:

- The plane wave test configuration of Richards et al. ${ }^{1}$ is used to provide simple and fast quantitative comparison of the non-reflective performance of methods based on buffer zones, characteristics, and far-field approximations.

*Research Fellow, Airbus Noise Technology Centre, J.Gill@soton.ac.uk.

${ }_{\dagger}$ Research Assistant, Airbus Noise Technology Centre

${ }^{\ddagger}$ Airbus Noise Technology Centre. Also Chair Professor, Department of Mechanical and Aerospace Engineering, The Hong Kong University of Science and Technology, Clear Water Bar, Kowloon, Hong Kong SAR, China. Associate Fellow, AIAA. 
- The plane wave test configuration is used to empirically optimize the shape and strength of the damping function in buffer zone methods and zonal CBC methods.

- A modified zonal CBC method is proposed for improved non-reflective performance.

- The proposed zonal CBC method is applied to a two-dimensional airfoil turbulence-interaction noise case and to a three-dimensional duct mode case.

\section{I.A. Methods to Prevent Reflections at Domain Boundaries}

One method to prevent reflections from the domain boundaries is to modify the edges of the simulation domain. Alterations such as making the domain edge normal to the incident waves and applying grid stretching, can significantly reduce reflections. However, in some situations, when studying duct acoustics or turbo-machinery for example, the geometric configuration may not allow for these changes to the domain boundaries. Therefore, non-reflective conditions must be applied at the domain edges. Three major categories of non-reflective conditions are those based on asymptotic far-field solutions, characteristic methods, and on buffer zone techniques. Comprehensive reviews of the different methods exist, by Colonius, ${ }^{2}$ and Tam, ${ }^{3}$ for example. Therefore, only a summary is given here.

Perhaps the most widely used non-reflective condition is based on characteristic methods. This method has been developed by authors such as Thompson, ${ }^{4,5}$ Poinsot and Lele, ${ }^{6}$ and Giles. ${ }^{7}$ The general idea of this approach is to perform a one-dimensional characteristic analysis at the domain boundaries. The value of characteristic waves entering the domain is set to zero to prevent reflections. However, because this method is formulated in one-dimension, it is less effective for waves which approach the boundary at oblique angles. Some authors, such as Yoo and $\mathrm{Im}^{8}$ have extended the approach to consider twodimensional problems, with some success, by including the transverse characteristic wave terms with a relaxation treatment. However, this approach was based on a low Mach number analysis. Liu and Vasilyev ${ }^{9}$ developed a nonlinear multidimensional characteristic approach that showed improvements in comparison to previous one-dimensional characteristic methods. CBC methods are usually applied to the edge of the computational domain. However, in some previous CAA studies, ${ }^{10,11}$ CBCs have been combined with buffer zone regions, which improves the performance for oblique waves. Sandberg and Sandham ${ }^{12}$ also proposed a zonal CBC approach, where the amplitude of the incoming characteristic wave is gradually ramped to zero at the outflow. This method was shown to significantly reduce reflections caused by outgoing vortical structures, since any reflected waves are contained within the zonal CBC region and cannot propagate back into the domain.

Non-reflecting conditions based on asymptotic far-field solutions were proposed by Bayliss and Turkel. ${ }^{13}$ Tam and Webb ${ }^{14}$ also developed this radiation condition, which assumes that acoustic waves radiate spherically from a point source in a uniform meanflow. This assumption allows alternative governing equations to be solved in a region at the edges of the domain. Tam and Dong ${ }^{15}$ extended this technique to consider weakly non-uniform meanflows, but limitations still exist for complex flow regimes.

Non-reflective conditions based on buffer zone techniques attempt to damp the flow-field to a prescribed value at the domain edges. This prevents waves from reaching the edges of the domain and thus prevents them from causing reflections. The domain is usually extended to include a buffer region in which the damping occurs. The damping can be achieved via numerical damping, ${ }^{1,11,16}$ grid stretching, ${ }^{17}$ or by accelerating the flow to supersonic speeds at the boundary, which removes the need for a non-reflective condition. ${ }^{18}$ The buffer zone method can provide good performance and is easy to implement, but the flow-field within the buffer zone itself is non-physical, and the extension of the domain can increase computational expense. The amount of damping is usually ramped up from zero in the domain to a prescribed amount at the edge of the simulation. Therefore, as with some other non-reflective boundary methods, buffer zones contain parameters which must be empirically tuned to optimize their performance. These parameters typically control the shape and strength of the damping ramp function.

A variation of the buffer zone method is known as the perfectly matched layer (PML). In this technique, a different formulation of the damping layer is solved in the buffer region, such that any reflections due to the damping process are avoided. In other buffer zone approaches, reflections can occur as a result of the damping process if the damping is too abrupt. The PML method was first formulated by Berenger ${ }^{19}$ and first used for CAA simulations by $\mathrm{Hu} .{ }^{20}$ Previous tests have found good results when using the PML method to solve the LEE, although it is difficult to apply to simulations using the Navier-Stokes equations. 
Some previous studies have assessed the relative performance of different non-reflective boundary conditions. Hixon et al. ${ }^{21}$ compared the performance of the characteristic methods of Thompson ${ }^{4}$ and Giles ${ }^{7}$ with the far-field condition of Tam and Webb ${ }^{14}$ when simulating a monopole in a uniform meanflow, and found the best performance with the Tam and Webb method. Bogey and Bailly ${ }^{22}$ showed that the performance of the Tam and Dong ${ }^{15}$ condition when considering both acoustic and vortical disturbances could be improved with the addition of a buffer zone. Richards et al. ${ }^{1}$ compared the performance of explicit and implicit buffer zones, the method of Freund et al. ${ }^{18}$ the PML condition of $\mathrm{Hu},{ }^{23}$ and Thompson's characteristic boundary condition. ${ }^{4,5}$ It was found that buffer zones using an explicit damping method gave the best performance. However, Richards et al. ${ }^{1}$ did not attempt to optimize the different techniques being used. For example, the strength and shape of the damping function in the implicit and explicit buffer techniques was not varied. Fosso et ll. $^{24}$ compared the performance of three characteristic techniques with the Tam and Dong ${ }^{15}$ condition when simulating acoustic propagation and vortex convection in uniform and non-uniform meanflow. It was found that for purely acoustic problems the Tam and Dong condition performed best, while the characteristic methods gave better results when considering vortical waves. However all methods suffered performance losses when considering non-uniform meanflow.

The previous comparisons of non-reflective techniques have shown that there is no single ideal method for removing reflections in all circumstances. Richards et al. showed that performance is dependent on the incident wave angle and frequency, the Mach number, the size of the non-reflective zone (if the method requires one), and any tunable parameters to set the shape of damping functions. Bogey and Bailly ${ }^{22}$ showed that performance can also depend on the type of wave being considered (eg: acoustic, vortical or entropic). With the exception of the study by Richards et al. ${ }^{1}$ most previous comparisons of non-reflective conditions have been largely qualitative in nature. However, although Richards et al. make a thorough quantitative comparison, the tunable parameters in the buffer zone approach are not optimized. Additionally, the explicit buffer zone, which is shown to be the most effective method by Richards et al., ${ }^{1}$ does not appear to be a generic solution for all cases. This is discussed in Section IV.

The current work evaluates and optimizes the performance of different non-reflective boundary methods for waves with varying frequency and incident angle. The aim is to find a generalized method which can perform adequately in a variety of circumstances. Such a condition is needed to allow simulation of turbomachinery cases such as airfoil turbulence-interaction noise simulations, for example, where acoustic and vortical waves will impact domain boundaries with a variety of frequencies and wave angles. Additionally, those boundaries cannot always be be moved or modified due to the specified geometry. Where non-reflective techniques contain tuneable parameters, attempts are made in the current work to optimize these for the best overall performance. To enable this comparison, a computational approach is adopted that is similar to Richards et al. ${ }^{1}$ Acoustic plane waves travel through an outgoing domain boundary, and the reflected wave is measured using Wilson' ${ }^{25}$ wavesplitting technique. The computational method is detailed in Section III.

In addition to comparing existing techniques, a modified zonal $\mathrm{CBC}$ method is proposed and evaluated. Following comparison with the various non-reflective approaches, the effectiveness of the proposed method is demonstrated on an airfoil turbulence-interaction noise case (which involves both acoustic and vortical wave interactions), and on a duct mode case.

\section{Description of Non-Reflective Conditions}

This section describes the non-reflective conditions that are tested in the current work. Testing all methods given in the literature is not feasible. Therefore, this study has chosen implementations from three of the main categories of non-reflective boundary condition: buffer zone, characteristic, and far-field approximation methods.

These boundary conditions are applicable to three-dimensional problems. For brevity they will be outlined for the two-dimensional Linearised Euler Equations which are given by

$$
\frac{\partial \boldsymbol{U}}{\partial t}+[\boldsymbol{A}] \frac{\partial \boldsymbol{U}}{\partial x}+[\boldsymbol{B}] \frac{\partial \boldsymbol{U}}{\partial y}=0,
$$

where $\boldsymbol{U}$ is the vector containing the disturbed primitive variables, $[\boldsymbol{A}]$ and $[\boldsymbol{B}]$ are the flux Jacobian matricies. 


\section{II.A. Buffer Zone Damping}

Richards et al. ${ }^{1}$ previously found that a buffer zone technique using explicit damping gave the best performance across a range of wave angles and frequencies. Therefore, this technique is tested in the current study.

The explicit buffer zone technique applies a direct forcing to the solution field, i.e.

$$
\hat{\boldsymbol{U}}^{n+1}=\boldsymbol{U}^{\boldsymbol{n}+\mathbf{1}}-\sigma(x)\left(\boldsymbol{U}^{\boldsymbol{n}+\mathbf{1}}-\boldsymbol{U}_{\text {target }}\right) .
$$

where $\boldsymbol{U}^{\boldsymbol{n}+\boldsymbol{1}}$ is the solution vector at the end of each time step, $\boldsymbol{U}_{\text {target }}$ is the desired target value (which is generally set to 0 when solving LEE), $\hat{\boldsymbol{U}}^{n+1}$ is the solution vector after the buffer zone treatment, and $\sigma(x)$ is the damping function defined as

$$
\sigma(x)=\alpha\left(1-\frac{L-x}{L}\right)^{\beta},
$$

where $\alpha$ is the maximum strength of the damping, $\beta$ sets the shape of the damping curve, $L$ is the length of the buffer zone region, and $x$ is the distance from the inner boundary of the buffer zone. Richards et al. set $\alpha=1$ and $\beta=3$ in the majority of tests. These tuneable parameters are optimized in Section $\mathrm{V}$ before comparisons with other non-reflective conditions are made.

In the current work it has been found that the performance of the explicit buffer zone method can vary across different test cases. This is discussed in Section IV. Therefore, an implicit buffer zone method is also tested as a potential improvement. In the implicit method, the damping is included as a source term on the right hand side of the governing equations, such that the LEE equations become

$$
\frac{\partial \boldsymbol{U}}{\partial t}+[\boldsymbol{A}] \frac{\partial \boldsymbol{U}}{\partial x}+[\boldsymbol{B}] \frac{\partial \boldsymbol{U}}{\partial y}=\sigma(x) \boldsymbol{U}
$$

where $\sigma(x)$ is given by Equation 3. Some authors, such as Kim et al. ${ }^{26}$ have used buffer zone profiles based upon cosine shapes as opposed to the polynomial function given in Equation 3. Both cosine and polynomial functions are tested in the current work.

\section{II.B. Characteristic Outflow}

There are various implementations for characteristic non-reflecting boundary conditions (CBCs). Therefore, in this section only a general outline of the method is given. Thompson ${ }^{4,5}$ described a locally one-dimensional inviscid CBC for the Euler equations. This was applied to the Navier-Stokes equations by Poinsot and Lele. ${ }^{6}$ The CBC described by Thompson is tested in the current work.

The governing equation given by Equation 1 can be rewritten as

$$
\frac{\partial \boldsymbol{U}}{\partial t}+[\boldsymbol{A}] \frac{\partial \boldsymbol{U}}{\partial x}=-[\boldsymbol{B}] \frac{\partial \boldsymbol{U}}{\partial y}
$$

This formulation considers the $y$-derivatives as a source term. By diagonalising the flux Jacobian this system can be decomposed into its one-dimensional characteristic modes. The diagonal term is defined as $\Lambda$ and is related to the flux Jacobian $\boldsymbol{A}$ by the matrix $\boldsymbol{P}$ and its inverse $\boldsymbol{P}^{-1}$ by

$$
\boldsymbol{\Lambda}=\boldsymbol{P}^{-1} \boldsymbol{A P}, \partial \boldsymbol{R}=\boldsymbol{P}^{-1} \boldsymbol{U},
$$

and the LEE equations can therefore be expressed in the following characteristic form,

$$
\frac{\partial \boldsymbol{R}}{\partial t}+\Lambda \frac{\partial \boldsymbol{R}}{\partial x}=\mathcal{T}
$$

The characteristics $(\mathcal{L})$ are defined as,

$$
\mathcal{L}=\boldsymbol{\Lambda} \frac{\partial \boldsymbol{R}}{\partial x}
$$

and the source terms on the right hand side are typically decomposed into viscous $\left(\mathcal{S}_{v}\right)$ and transverse terms $(\mathcal{T})$. However, in the current implementation for LEE the viscous terms are not present. The characteristic 
waves are entropy, vorticity and acoustic modes. The convection of the modes is determined by the wave speed contained in the diagonal of the vector $\Lambda$. For a non-reflecting outflow condition, the time derivative at the boundary face is modified to ensure that the upstream travelling acoustic wave has a zero amplitude. Once the non-reflective condition is set in the characteristic formulation, the flux in the $x$-direction can be recalculated to provide the final prediction of the time derivative. For non-linear computations this method can generate unphysical results where the mean outflow pressure diverges. ${ }^{6,27}$ This problem is not present in the LEE solver because the mean pressure is constant. The outlined method considers only characteristics travelling along the spatial coordinate axis. Kim and $\mathrm{Lee}^{27}$ derived generalised CBCs for the Euler and Navier-Stokes equations which work in the computational space. This is convenient since outflow boundaries may not always be aligned with the physical coordinate system.

Yoo and $\mathrm{Im}^{8}$ proposed an extension to the one-dimensional CBC method that accounts for transverse characteristic terms, in order to provide better performance for oblique waves. A modified implementation of this is tested in the current work. To account for the transverse terms, Yoo and Im proposed to set the value of $\mathcal{L}_{5}$ as

$$
\mathcal{L}_{5}=(A-B) * \mathcal{T}_{5}
$$

where $\mathcal{T}_{5}$ represents the transverse terms. Yoo and $\operatorname{Im}^{8}$ set $A=1$ and show that the parameter $B$ must scale with the Mach number. However, the most appropriate values of $A$ and $B$ remain a subject of discussion, particularly because the analysis of Yoo and $\operatorname{Im}^{8}$ was based on low Mach number analysis, and is therefore not valid at higher speeds. In the current work, a parameter study was made to empirically find the most appropriate values of $A$ and $B$. Therefore, the current work sets the value of $L_{5}$ as

$$
\mathcal{L}_{5}=\max (0.0,(A-B M)) * \mathcal{T}_{5}
$$

where $A=0.3$ and $B=0.7$, and $M$ is the Mach number. Further detail is given in Section VI.A.

\section{II.C. Far-Field Non-Reflective Condition}

In previous tests of non-reflective conditions the far-field radiation condition of Tam and Webb, ${ }^{14}$ and Tam and Dong ${ }^{15}$ has performed well. Therefore, the method proposed by Tam and Webb ${ }^{14}$ will be tested in this work. Tam and Dong ${ }^{15}$ presented an extension of the method to weakly non-uniform meanflows. However, this had no effect in the plane wave test case used here, so it is not included.

In the method of Tam and Webb, ${ }^{14}$ alternative governing equations are solved at the edges of the domain, that are based on the radiation of an acoustic wave from a single point. The boundary condition is given by solving

$$
\left(\frac{1}{V(\theta)} \frac{\partial}{\partial t}+\frac{\partial}{\partial r_{0}}+\frac{1}{2 r_{0}}\right)\left[\begin{array}{l}
\rho \\
u \\
v \\
p
\end{array}\right]=0
$$

where $r_{0}$ is the distance to the observer, $\theta$ is the angular co-ordinate of the boundary point, $V(\theta)=$ $a_{0}\left(M \cos (\theta)+\sqrt{1-M^{2} \sin ^{2} \theta}\right)$, and $a_{0}$ is the speed of sound.

The Tam and Webb condition is intended for situations involving an acoustic point source. In the plane wave test case used in this paper, this is not the case. To make this method compatible without modification, the angle of the wave $\theta$ is used as an input, and it is assumed that the domain edge is a large distance from a point source, such that the wave can be approximated as a plane wave. Therefore, $r_{0}=1 \times 10^{6} \mathrm{~m}$ was used. The requirement that the angle of the incoming wave is a known quantity, represents a limitation of the Tam and Webb boundary condition, as it prevents use in generic situations where this angle is not known.

\section{II.D. Summary of Methods}

The methods that are compared in this study are:

- Explicit and implicit formulations of the buffer zone method, as previously tested by Richards et al. ${ }^{1}$ 
- Thompson's CBC, ${ }^{4,5}$ referred to here at the local CBC method.

- The zonal CBC method proposed by Sandberg and Sandham. ${ }^{12}$

- A new zonal transverse characteristic boundary condition (TCBC), which combines the zonal CBC method with the inclusion of transverse characteristic terms.

- The far-field radiation condition of Tam and Webb. ${ }^{14,15}$

\section{Computational Method}

The computational method in the current study uses a high-order finite difference code to solve the LEEs, which has been used by several previous authors (see Refs. ${ }^{28,29,30,31}$ for example). A sixth-order prefactored compact spatial discretization scheme ${ }^{32}$ is used alongside a fourth-order explicit temporal scheme. ${ }^{20}$ Tenthorder explicit filtering is used to provide numerical stability. The numerical methods ensure low-dispersion and low-dissipation errors and therefore can propagate vortical and acoustic waves with high accuracy.

A configuration similar to Richards et al. ${ }^{1}$ is used to test the performance of the non-reflective boundary conditions for different wave frequencies, angles, and Mach numbers. The configuration uses a three block Cartesian rectangular grid. Block one is used to generate disturbances, block two contains the main domain, and block three contains the outflow region. The upper and lower faces of the domain are set to a periodic boundary condition to reduce the required cell count. A constant grid spacing of $0.025 \mathrm{~m}$ is used throughout this study, which ensures that acoustic waves are resolved by at least 12 points per wavelength. A schematic of the configuration is shown in Figure 1.

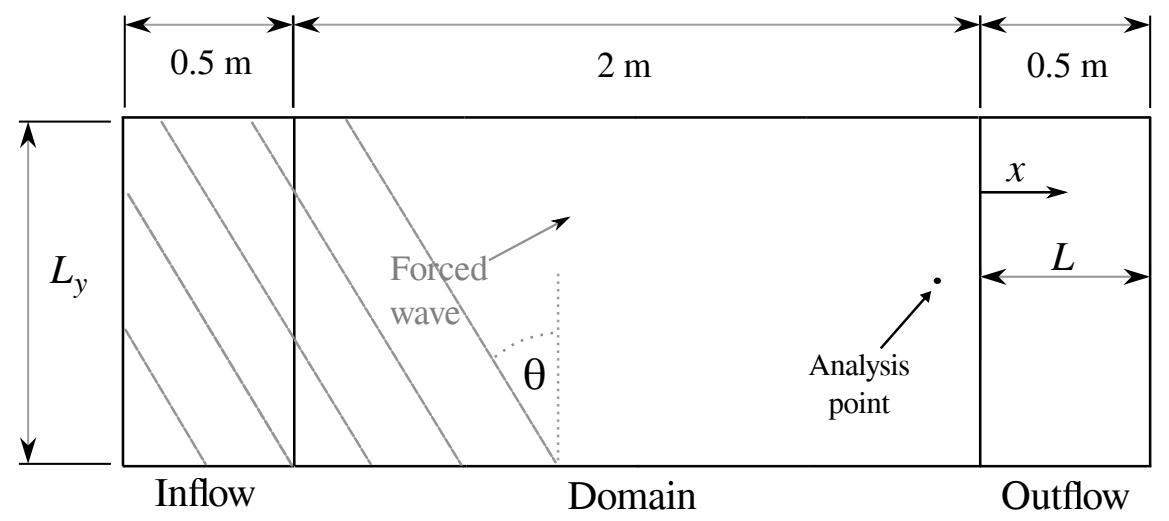

Figure 1. A Schematic of the non-reflective boundary condition test case.

\section{III.A. Definition of Input Waves}

Two-dimensional plane waves are forced with varying frequency and cut on ratio. In complex notation, the pressure wave is given as

$$
p(x, y, t)=89 e^{i\left(\omega t-k_{x} x-k_{y} y\right)}
$$

where $p$ is the acoustic pressure, $\omega$ is the angular frequency, $k_{x}$ and $k_{y}$ are the wavenumber components of the wave, and the amplitude of the wave is set to $89 \mathrm{~Pa}$ for consistency with Richards et al. ${ }^{1}$ The ratio $k_{x} / k_{y}$ is defined by the cut-on ratio $\xi$, as

$$
\frac{k_{x}}{k_{y}}=\frac{-M \xi+\sqrt{\xi^{2}-1}}{\sqrt{1-M^{2}}}
$$

where $M$ is the freestream Mach number. The angular frequency is given by $\omega=\xi k_{y} \sqrt{1-M^{2}}$. The waves are forced at the inflow to the simulation using an implicit buffer technique, where the value of $\boldsymbol{U}_{\text {target }}$ is set to equal the unsteady amplitude of the forced wave. Because the domain is small and periodic, specific values of $\omega$ and $\xi$ must be chosen in order to obey the periodicity condition. To allow a wider variety of 
wave inputs, domains of different heights $L_{y}$ are used. Therefore, in each simulation desired values of $\xi$ and $L_{y}$ are chosen, and the value of $\omega$ is found using the described relations.

\section{III.B. Quantifying the Reflections}

Wilson's wavesplitting technique ${ }^{25}$ is used to quantify the reflections, as detailed by Richards et al. ${ }^{1}$ Using this technique, the magnitude of the upstream and downstream waves can be quantified with

$$
p_{\omega, k_{y}}^{d s / u s}=0.5\left(p_{\omega, k_{y}} \pm \frac{\xi}{\sqrt{\xi^{2}-1}} u_{\omega, k_{y}} \pm \frac{M}{\sqrt{\xi^{2}-1} \sqrt{1-M^{2}}} v_{\omega, k_{y}}\right) .
$$

In this study, $p, u$, and $v$ are recorded in the domain at a single point just upstream of the outflow buffer zone, as shown in Figure 1.

\section{Initial Tests of The Buffer Zone Method}

As an initial test of the performance of the explicit buffer zone method, the reflections were measured from the explicit buffer zone implementation using $\alpha=1$ and $\beta=3$ when using three different timestep sizes. In this test, $M=0$ and $L_{y}=1$. The variation of reflections with $\xi$ and $\theta$ is plotted in Figure 2.
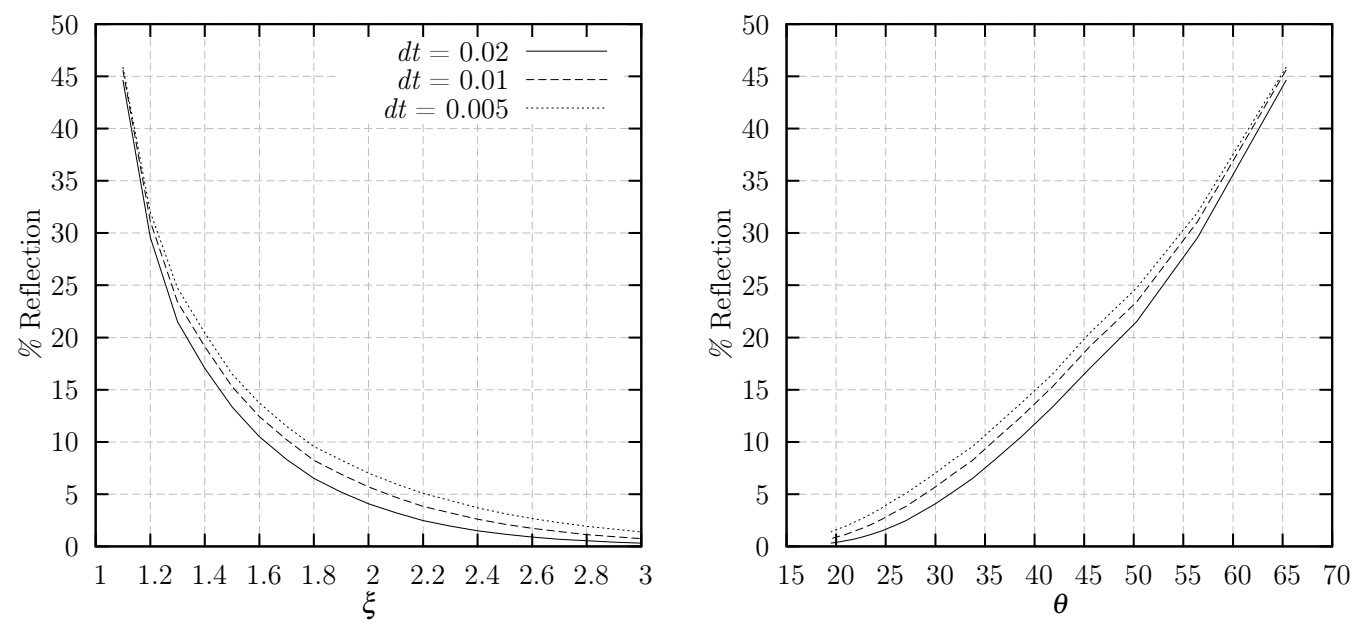

Figure 2. Variation of reflections with $\xi$ and $\theta$ for non-dimensional timesteps of $d t=0.01,0.005$, and 0.0025 .

Figure 2 shows that the performance of the explicit buffer zone is dependent on the timestep of the simulation. When the timestep is decreased, the resulting reflections are increased. This is explained by the explicit nature of the this buffer zone. If the timestep is smaller, the damping will be applied a larger number of times as the wave propagates through the buffer zone. In Figure 2 this is causing the damping to be too strong, which in turn increases the amount of numerical reflection. Therefore, the explicit buffer zone is not a good choice for a generic buffer condition because it is timestep-dependent. In the remainder of this paper, the explicit buffer zone method is discarded in favor of an implicit buffer zone approach.

In the implicit buffer zone approach, the damping is independent of the timestep size. Richards et $a l .{ }^{1}$ also tested an implicit buffer zone and found that performance was poor in comparison to the explicit method. However, no attempt was made to optimize $\alpha$ and $\beta$, which were respectively fixed at 1 and 3 in the analysis. In this work, the tuneable parameters are optimized to improve the performance.

To verify that the current study produces similar results to Richards et al. the reflections were measured for waves in a $M=0$ flow, when using the explicit and implicit buffer zones, and Thompson's CBC. The trend of reflections with varying cut-on ratio is presented in Figure 3, for both the current results and those from Richards et al..$^{1}$

The general behavior of the numerical reflections with $\xi$ that is shown in Figure 3 agrees with the findings of Richards et al. ${ }^{1}$ As the cut-on ratio $\xi$ is increased, the non-reflective performance is reduced. For the implicit buffer zone and characteristic methods, the results are similar to those presented by Richards et al. 

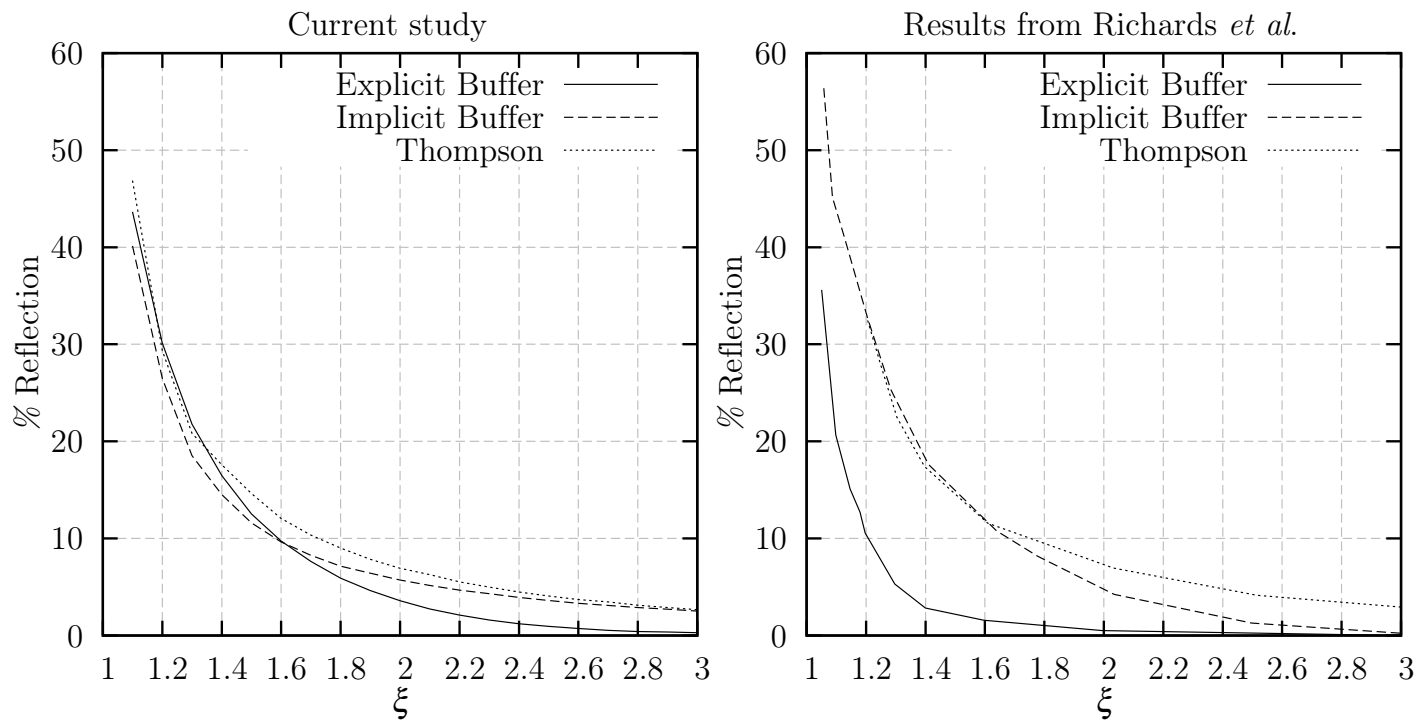

Figure 3. Variation of reflections with $\xi$ for the explicit and implicit buffer methods, and for the characteristic method.

However, the reflections found here using the explicit buffer zone are larger than found by Richards et al. for all values of $\xi$. The cause of this discrepancy is not known. However, Richards et al. do not state the simulation timestep that was used. The use of a different timestep may explain this discrepancy. 


\section{Optimization of Tuneable Parameters}

\section{V.A. Buffer Zone Optimization}

Before a comparison between the different non-reflecting methods is made, each boundary condition should function at optimal conditions. The buffer zone method uses two empirical parameters to set the shape and strength of the damping, which are $\alpha$ and $\beta$. An optimum value of $\alpha$ and $\beta$ that provides good performances for various incident waves at various flow speeds is desired. To find an optimum value, a parameter study was made which varied the values of $\alpha$ and $\beta$ in a large number of independent simulations, and measured the resulting reflections. Values of $\alpha$ were varied between 1 and 20. These values were respectively used for an implicit buffer zone forcing method by Richards et al. ${ }^{1}$ and Collis and Lele. ${ }^{11}$ Values of $\beta$ were varied between 0.25 and 5 . To ensure the chosen parameters give a good performance in a variety of situations, the study was made at two Mach numbers of $M=0$ and 0.5 , and using three values of the cut-on ratio $\xi=1.2$, 2 , and 3. In total this gave 2400 individual cases. However the fast and simple nature of the plane wave test configuration allowed the test to be run on a single core of a desktop computer in approximately 3 hours.

Contours of varying percentage reflection with $\alpha$ and $\beta$ are shown in Figure 4 for varying Mach number and cut-on ratio. Figure 4 shows that for each value of $\xi$ the reflection can be significantly affected by varying $\alpha$ and $\beta$. At $\xi=1.2$, the lowest reflection is given for $\alpha \approx 1$ and $\beta \approx 0.25$, which is the limit of the tested parameter range. The amplitude of the reflected wave is varied by up to $50 \%$ due to changes in $\alpha$ and $\beta$, and is therefore sensitive to the tuneable parameters. At $\xi=2$ there is still a clear minimum value at $\alpha \approx 7$ and $\beta \approx 2$. However, the reflection amplitude is less sensitive to the tuneable parameters, and varys by no more than $12 \%$ at $M=0$. At $\xi=3$ the reflections appear to be insensitive to the tuneable parameters, except for high values of $\alpha$ or $\beta$. While the reflection amplitude at each cut-on mode is dependent on the Mach number, the location of the optimum combination of tunable parameters appears to be insensitive to Mach number.

Figure 4 suggests that the tuneable parameters should be optimized to give the most benefit to low values of $\xi$, since these acutely angled waves show the highest sensitivity to changes in $\alpha$ and $\beta$. Therefore, four combinations of $\alpha$ and $\beta$ were chosen for testing at a wider range of $\xi$ values. These combinations are given in Table 1.

\begin{tabular}{ccc}
\hline Combination & $\alpha$ & $\beta$ \\
\hline 1 & 1 & 0.5 \\
2 & 2 & 1 \\
3 & 4 & 1.5 \\
4 & 6 & 2 \\
\hline
\end{tabular}

Table 1. Combinations of $\alpha$ and $\beta$ chosen for additional testing.

Figure 5 shows the variation of reflection amplitude with $\xi$, for the combinations of parameters given in Table 1. This comparison has been made at $M=0$ (since the optimization appears to be insensitive to Mach number), and used a domain height of $L_{y}=1 \mathrm{~m}$. Figure 5 reveals a behavior that was not seen in the initial parameter study; low combinations of $\alpha$ and $\beta$ improve the non-reflective performance at $\xi=1.1-1.2$ but degrade the performance at $\xi=1.3-1.8$. This degradation represents waves which reflect from the domain edge and propagate back out of the buffer zone due to insufficient damping. While it is possible to justify any of the parameter combinations in Table 1, the fourth combination, $\alpha=6$ and $\beta=2$ was chosen as the optimized combination in the current study. This combination gives the best performance for wave angles up to $\theta=35^{\circ}$, and acceptable performance (up to $7 \%$ reflection amplitude) up to $\theta=45^{\circ}$. 

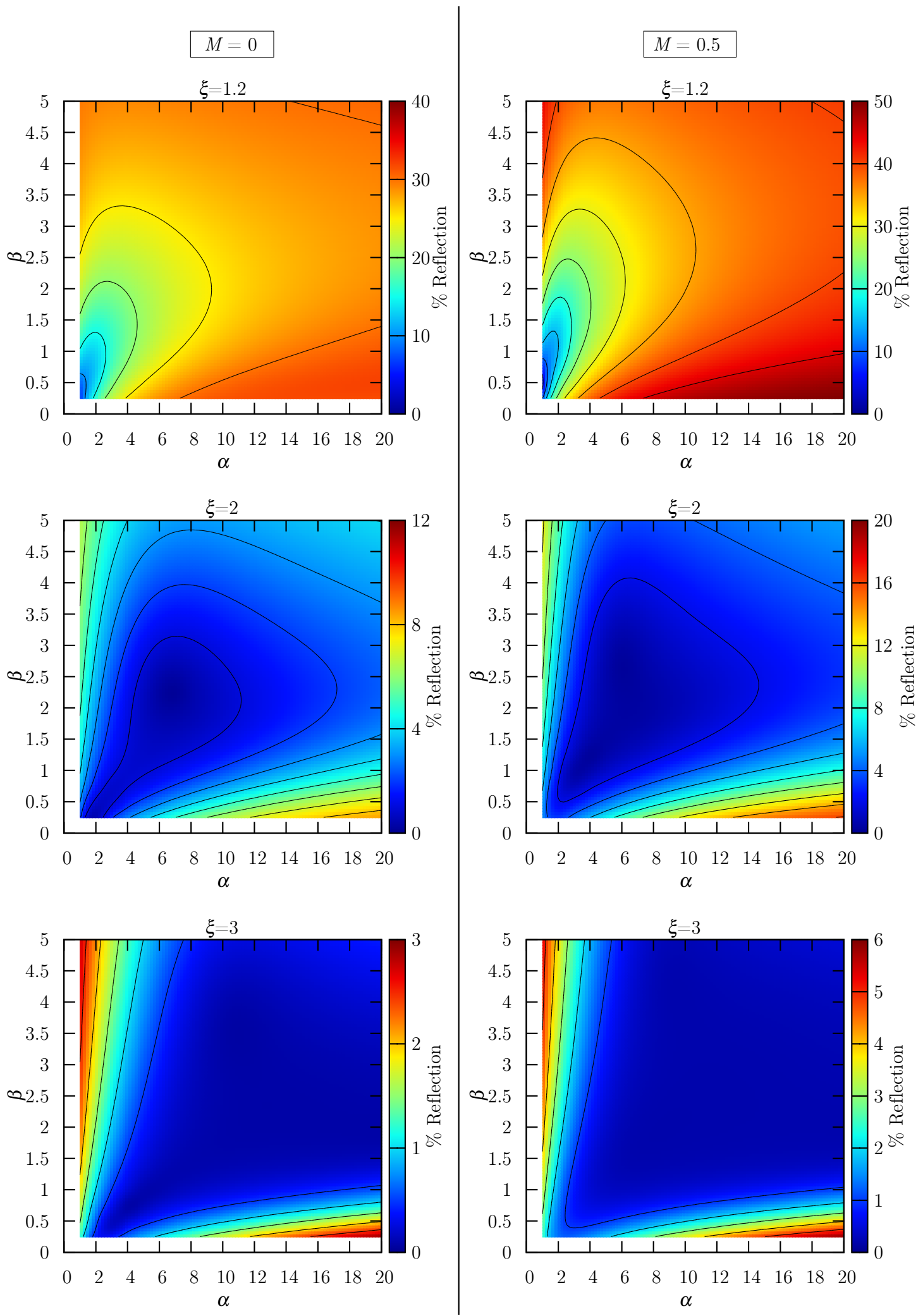

Figure 4. Numerical reflections with varying $\alpha, \beta, M$, and $\xi$. 

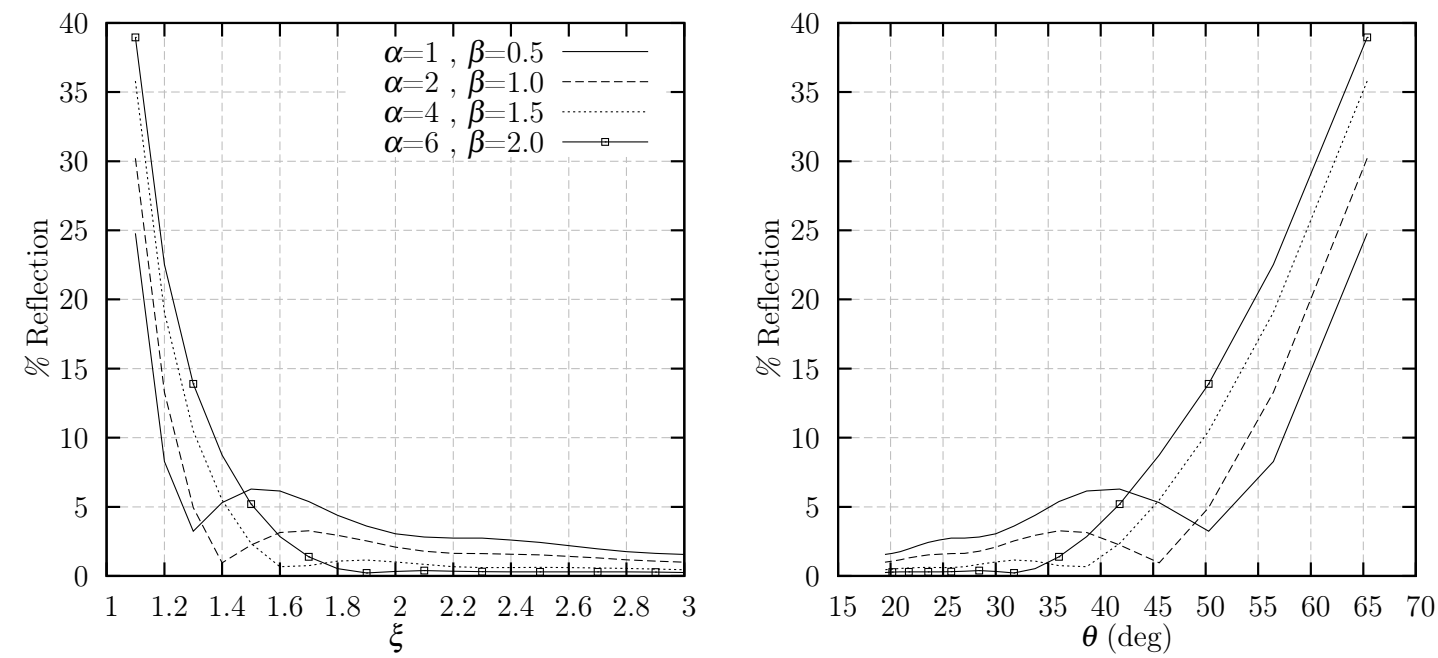

Figure 5. Numerical reflections with varying $\xi$ and $\theta$ for four different combinations of the tuneable parameters.

\section{V.B. Alternative Damping Shape Function}

Thus far, this study has considered a polynomial damping profile, as given by Equation 3. Some authors, such as Kim et al. ${ }^{26}$ for example, use an alternative profile based on a cosine function. The effect of a cosine profile is tested in this section.

The cosine damping profile that is used here can be written as

$$
\sigma(x)=\frac{\alpha}{2}(1+\cos (\pi(1-x / L))) .
$$

where $\alpha$ is a single tunable parameter which sets the strength of the damping. The previously described optimization parameter study was used to find an optimal value of $\alpha$, which was chosen to be $\alpha=4$.

Figure 6 compares the reflection amplitude with varying $\xi$ for the polynomial and cosine damping profiles. A similar performance is seen by both approaches. However in the range $1.6<\xi<2.2$ the polynomial profile gives reduced reflection amplitudes. Therefore, the polynomial function, using $\alpha=6$ and $\beta=2$, was chosen to compare with other non-reflective conditions in Section VI.

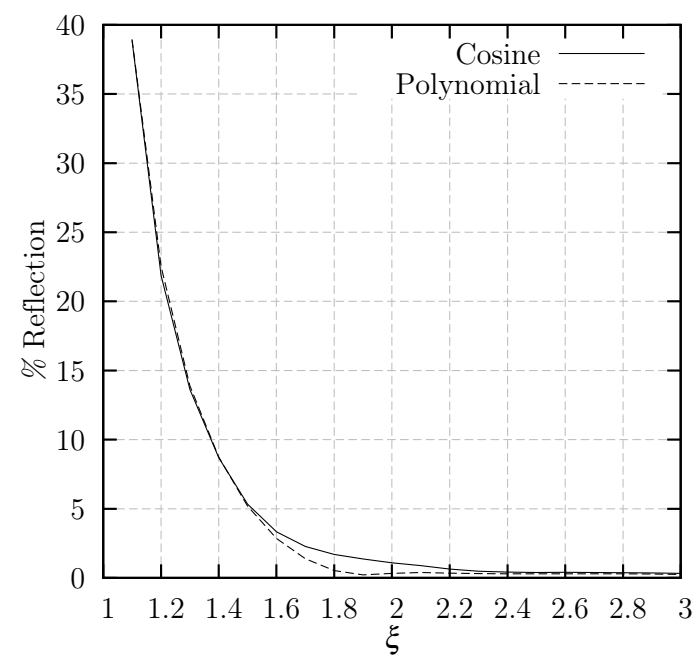

Figure 6. Comparison of reflection amplitude when using an implicit buffer zone method with cosine and polynomial damping functions. 


\section{V.C. Zonal CBC Optimization}

The zonal CBC method must also be optimized to obtain the best performance. While the original CBC method proposed by Thompson ${ }^{4,5}$ did not require optimization, the approach that is used here requires the shape of the zonal damping function to be defined. Similarly to the optimization of the buffer zone approach, a cosine damping function, and polynomial damping functions were tested. Polynomial functions were used as defined by Equation 3, with the $\alpha$ parameter removed, and with $\beta=1,2$, and 3 . The numerical reflections given by each damping function were measured for waves with various cut-on ratios. The different damping functions, and the corresponding numerical reflections, are shown in Figure 7.
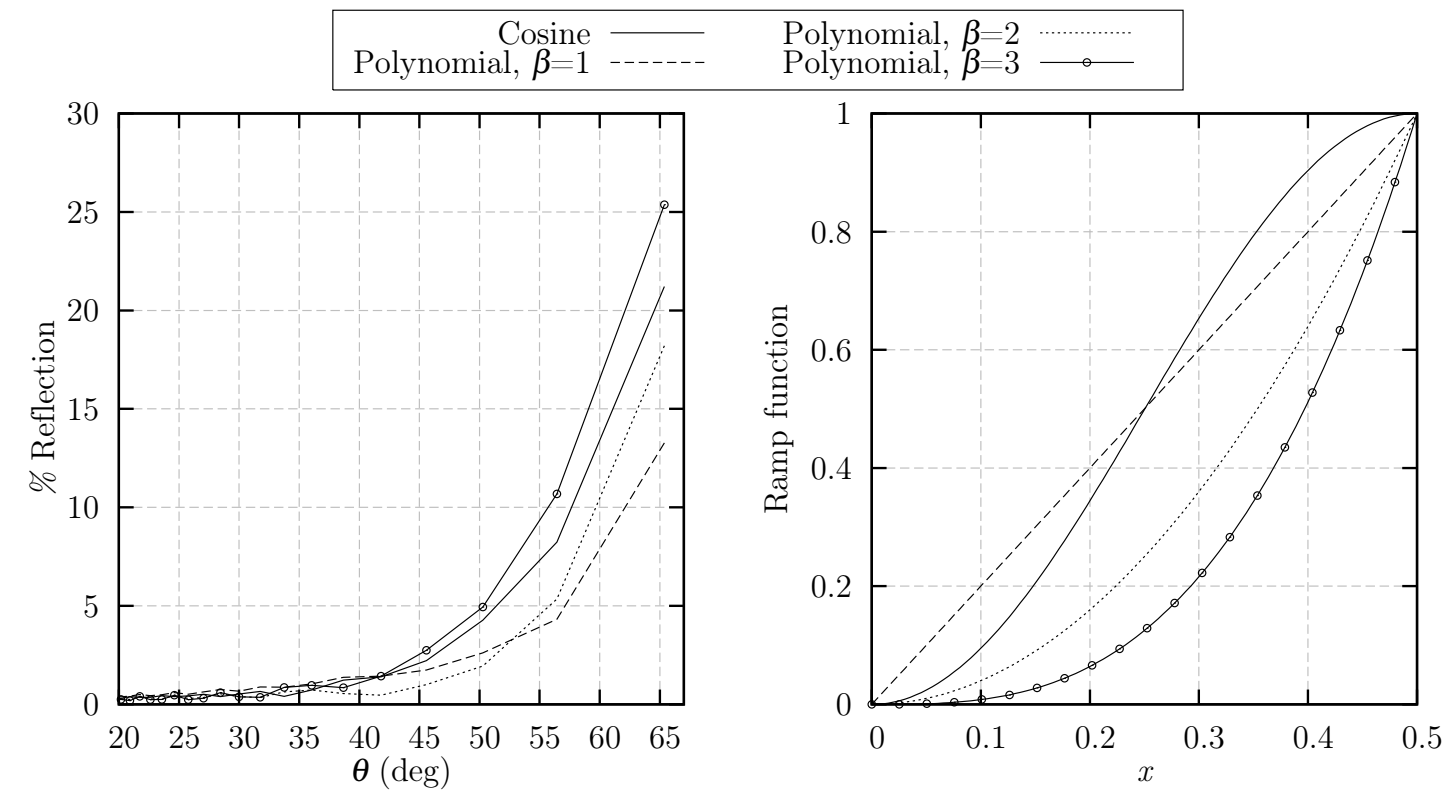

Figure 7. (Left) Numerical reflections with varying $\theta$ in $M=0$ flow, when using different zonal CBC ramping functions. (Right) Different ramping functions tested for the zonal CBC method.

Only small variations in non-reflective performance are shown by Figure 7, which suggests that the zonal CBC method is relatively insensitive to the shape of the damping function. However, the polynomial function with $\beta=2$ appears to offer reduced reflections in comparison to the other tests, for wave angles up to $\theta=50^{\circ}$. At higher wave angles a better performance is seen by the $\beta=1$ polynomial function. However, waves above $\theta=50^{\circ}$ are considered to be less common and therefore less important than wave angles below $\theta=50^{\circ}$. Therefore, the polynomial function with $\beta=2$ was chosen as the zonal CBC damping function in the remainder of this work. 


\section{Comparison of Non-Reflective Conditions}

This section compares the non-reflective performance of the buffer zone, Tam \& Webb, local CBC, and zonal CBC methods for waves with various cut-on ratio and for $M=0$ and $M=0.5$. Figure 8 shows the non-reflective performance with varying cut-on ratio $\xi$, and Figure 9 shows the performance with varying wave angle $\theta$.

At $M=0$, Figures 8 and 9 show that the least effective non-reflective boundary condition appears to be the local CBC approach. The local CBC approach gives a consistently higher reflection than the alternative methods at all tested cut-on ratios. The buffer zone approach is more effective, giving almost zero reflection for $\theta<35^{\circ}$. However, at angles above this threshold, the performance reduces such that a $38 \%$ reflection is measured for $\theta=65^{\circ}$. The zonal CBC method shows improved performance, with less than $5 \%$ reflection for waves below $\theta=50^{\circ}$. However, the best performance is given by the Tam \& Webb condition, which gives an almost zero reflection at all tested cut-on ratios. However, as previously discussed, the Tam \& Webb condition is less generic than the other tested methods, because it requires the wave angle to be known.

At $M=0.5$, the relative performance of the four approaches is altered in comparison to the $M=0$. results. At $M=0.5$, the worst performance is given by the buffer zone approach, and the local and zonal CBC methods show an improved performance. The local CBC method shows a maximum reflection of $7 \%$, while the zonal CBC method has a maximum of $1 \%$. Therefore, it appears that the non-reflective performance of $\mathrm{CBC}$ methods will improve with increasing Mach number. The best performance at $M=0.5$ is given by the Tam \& Webb condition, similarly to the results at $M=0$.

Figures 8 and 9 show that the most effective generic non-reflective method appears to the zonal CBC method. The performance of this method is close to the Tam \& Webb method at $M=0.5$, but less effective at $M=0$.
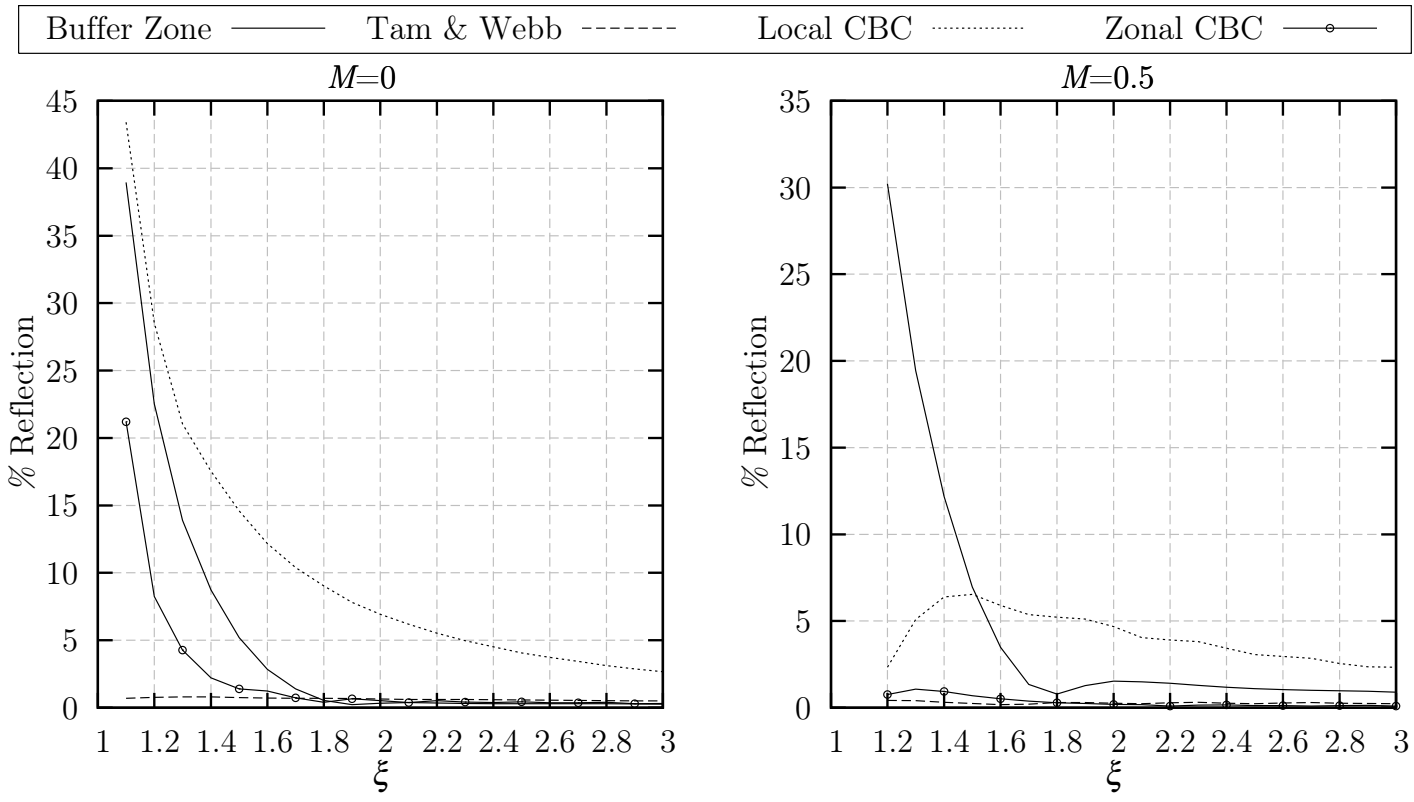

Figure 8. Numerical reflections with varying $\xi$ for $M=0$ and $M=0.5$ using different non-reflective conditions.

To investigate the behaviour of the zonal CBC method with increasing Mach number, Figure 10 compares the numerical reflections at various wave cut-on ratios for Mach numbers varying between $M=0$ and $M=0.3$. Figure 10 shows that the non-reflective performance consistently improves with increasing Mach number. As $M$ increases, the wave angle required to give a $5 \%$ reflection also increases, At $M=0.3$, even a wave with $\theta=83^{\circ}$ will cause only a $4 \%$ reflection. Therefore, it appears that the zonal CBC method will perform well (with numerical reflections of less than 5\%) for almost any wave if $M \geq 0.3$. 

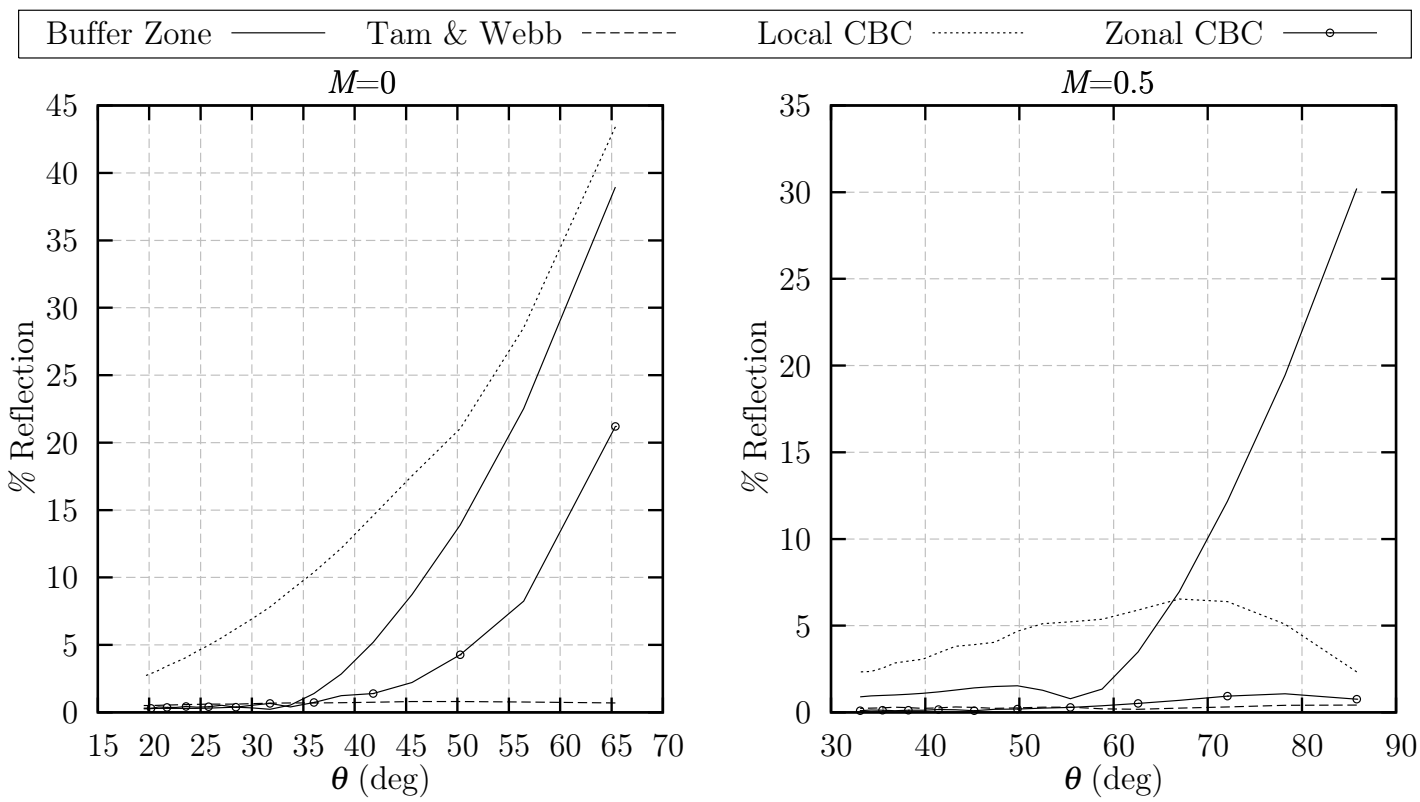

Figure 9. Numerical reflections with varying $\theta$ for $M=0$ and $M=0.5$ using different non-reflective conditions.

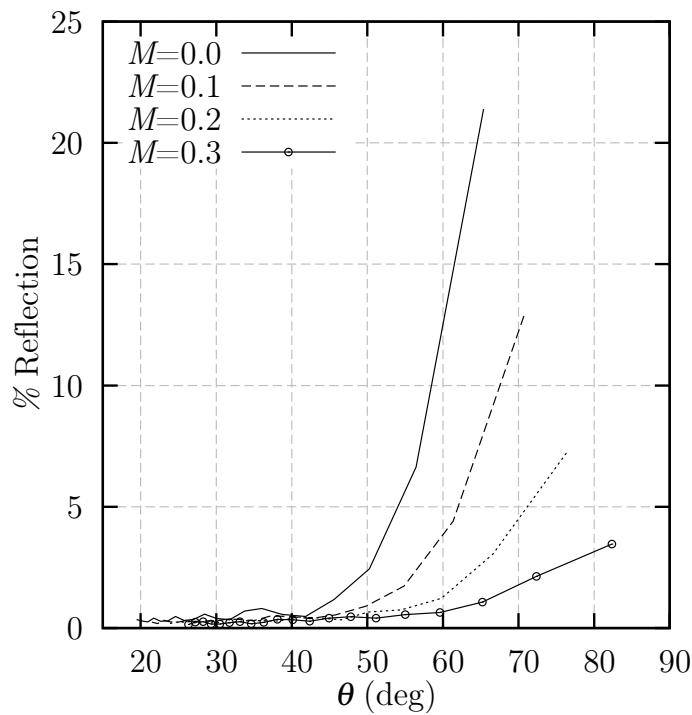

Figure 10. Numerical reflections with varying $\theta$ for $M=0$ to $M=0.3$ for the zonal CBC method. 
Discussions in this work have shown that non-reflective performance is dependent on Mach number and on the wave cut-on ratio (or wave angle). For methods which act over a zone, such as the buffer zone and the zonal CBC, Richards et al. ${ }^{1}$ also showed that performance will depend on the ratio of the wavelength to the size of the damping region. In order to study the non-reflective performance more thoroughly, Figure 11 shows contours of numerical reflections for the zonal CBC and buffer zone methods at $M=0$, for varying $\theta$ and for varying ratio $\lambda_{x} / L$, where $\lambda_{x}$ is the wavelength in the $x$-axis direction. Contour lines have also been plotted to show the combination of $\theta$ and $\lambda_{x} / L$ that will result in both $5 \%$ and $10 \%$ reflections.

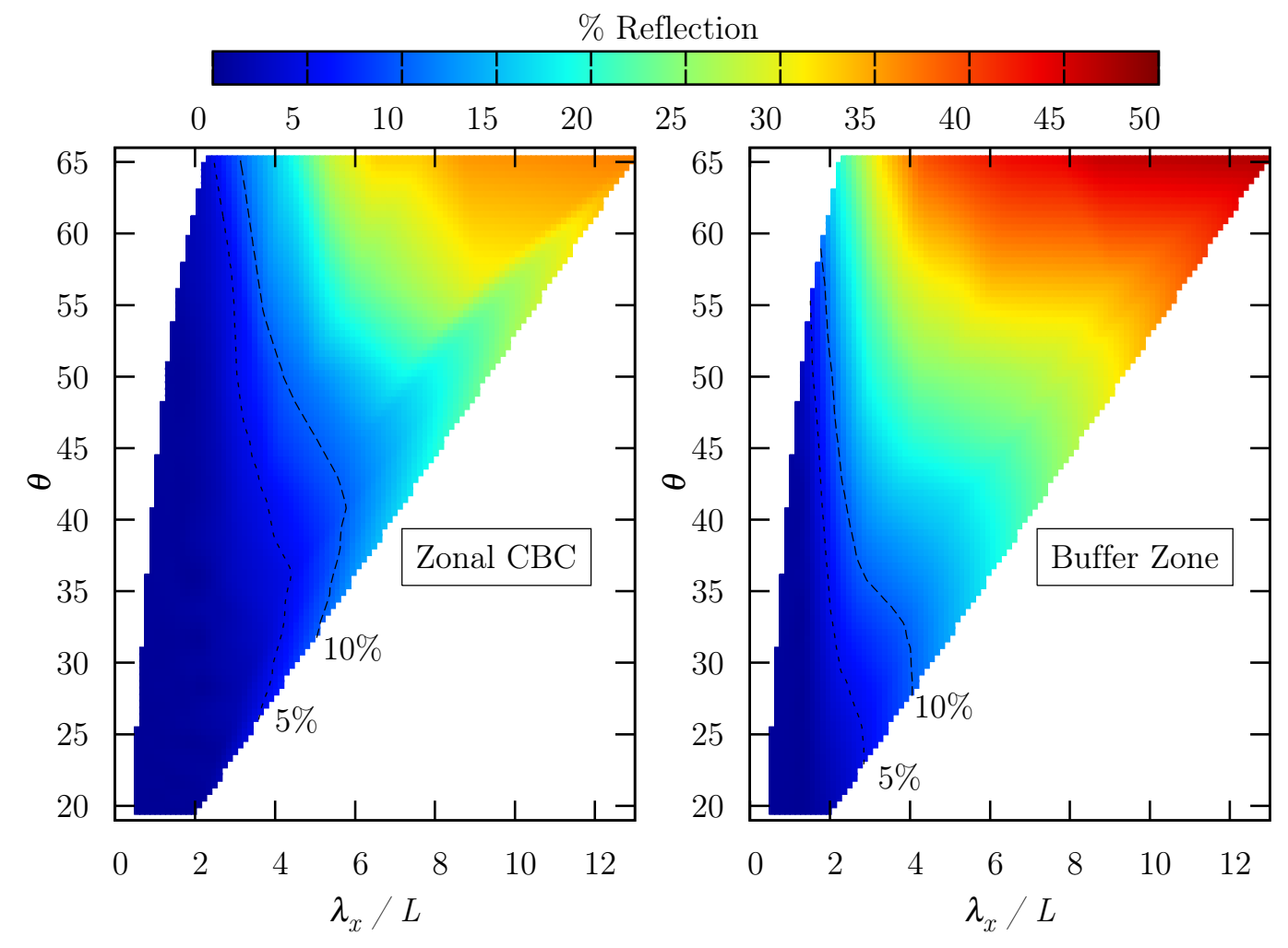

$\%$ Reflection

Figure 11. Contours of numerical reflection with varying $\lambda_{x} / L$ and $\theta$, for the buffer zone and zonal CBC methods.

Figure 11 shows that the performance of both the buffer zone and zonal CBC methods has a dependence on $\theta$ and $\lambda_{x} / L$. The worst performance for both methods is seen when both $\theta$ and $\lambda_{x} / L$ is high. As was shown in in Figures 8 and 9, the zonal CBC method appears to give a better performance than the buffer zone method throughout the range of tested parameters. Figure 11 shows that in order to achieve no more than $5 \%$ reflections at $\theta=35^{\circ}$ with the zonal CBC method, $\lambda_{x}$ must be no greater than 4 times the length of the buffer zone. At other values of $\theta$, the $\lambda_{x} / L$ requirement is reduced. The corresponding maximum $\lambda_{x} / L$ value for the buffer zone method occurs at $\theta=23^{\circ}$, and is equal to $\lambda_{x} / L=3$. Therefore, the zonal CBC method allows a smaller damping region to be used in comparison to the buffer zone method, for a given acoustic wavelength. 


\section{VI.A. Zonal TCBC method with transverse terms}

It was shown in Section VI that the best generic non-reflective condition that has been tested in the current study is the zonal CBC method. At high Mach number this method gives close to zero numerical reflections for most wave angles and wavelengths. However for $M<0.3$, the performance is less satisfactory. Numerical reflections of up to $22 \%$ have been measured at $M=0$ for waves with $\theta=65^{\circ}$. This section proposes a modified zonal CBC method in order to improve the non-reflective performance at low Mach number.

As discussed in Section II, Yoo and $\operatorname{Im}^{8}$ proposed to account for the transverse terms in a one-dimensional CBC method by using a relaxation technique to set the value of the incoming characteristic terms to depend on the transverse component $\mathcal{T}_{5}$, according to $\mathcal{L}_{5}=(A-B M) \mathcal{T}_{5}$, with $A=B=1$. In the current work, a parameter study was made to view the effect of different values of $A$ and $B$ when introducing the transverse characteristic terms. Figure 12 shows the measured reflection at varying Mach number, with values of $A$ varying between 0 and 1 , and for $B=1.0$ and 0.7. The results in Figure 12 are for a wave with $\xi=1.1$. However, the effect is repesentative of the behaviour at all $\xi$ values.
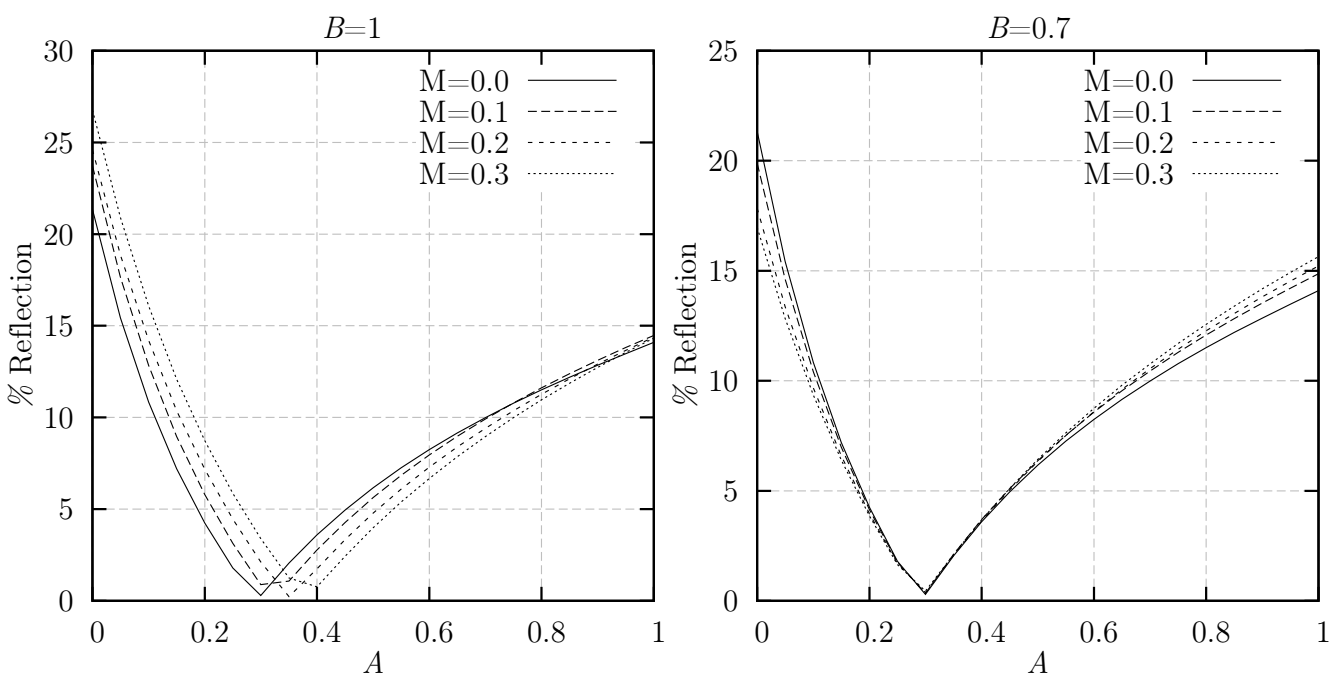

Figure 12. Numerical reflections for waves with $\xi=1.1$ at various Mach numbers, and with various values of $A$. Results are shown for $B=1$ (left) and for $B=0.7$ (right).

Figure 12 shows that the choice of $A$ has a significant effect on the numerical reflection. If $B=1$, $A=0$, and $M=0$ (i.e. the transverse terms are neglected since this makes $\mathcal{L}_{5}=0$ ) the reflection is $22 \%$. If $A$ is then changed to $A=1$ as suggested by Yoo and Im, the reflection is reduced to $14 \%$. However, significant improvements are seen by setting $A=0.3$, which appears to almost completely remove the numerical reflection. Similar behaviour is seen for other values of $M$ when $B=1$, with the exception that the minimum reflection value moves away from $A=0.3$ as $M$ increases.

The parameter study also highlighted the effects of variations in $B$. Figure 12 shows that if $B$ is changed from 1 to 0.7 , the reflection curves collapse more closely onto a single line. Differences are seen between studies at different Mach number when $A$ approaches either 1 or 0 . However, the collapse is good in the region where numerical reflections are smallest. It should be noted that various values of $B$ were tested, and that results for $B=0.7$ are shown here because this value gives the best collapse of data.

Significant improvement in the numerical reflections at various Mach numbers has been found if the transverse terms are included in the $\mathrm{CBC}$ method with $A=0.3$ and $B=0.7$. The final consideration is that the transverse terms need implementing only at low values of Mach number. This is because the performance of the characteristic method appears to improve with increasing Mach number. Therefore, the final expression for setting the target value of $\mathcal{L}_{5}$ was chosen to be

$$
\mathcal{L}_{5}=\max (0.0,(A-B M)) \mathcal{T}_{5}
$$

This expression ensures that the transverse terms are used only when $M \leq 0.3 / 0.7$. The proposed method is referred to as the zonal TCBC method in the remainder of this work. 


\section{VI.B. Performance of the zonal TCBC method}

In this section the performance of the zonal TCBC and zonal CBC methods is compared. Figure 13 compares the numerical reflections for waves at different angles $\theta$, and at different Mach numbers. Low speed Mach numbers are tested because the zonal TCBC and CBC methods are identical at high Mach number. Figure 13 shows that the TCBC method produces significantly smaller reflections than the CBC method at all tested wave angles and Mach numbers. The largest numerical reflection given by the TCBC method is approximately $1 \%$, and the reflection amount is almost constant with wave angle. Therefore, it appears that the inclusion of the transverse terms according to Equation 16 gives a significant improvement in non-reflective performance.

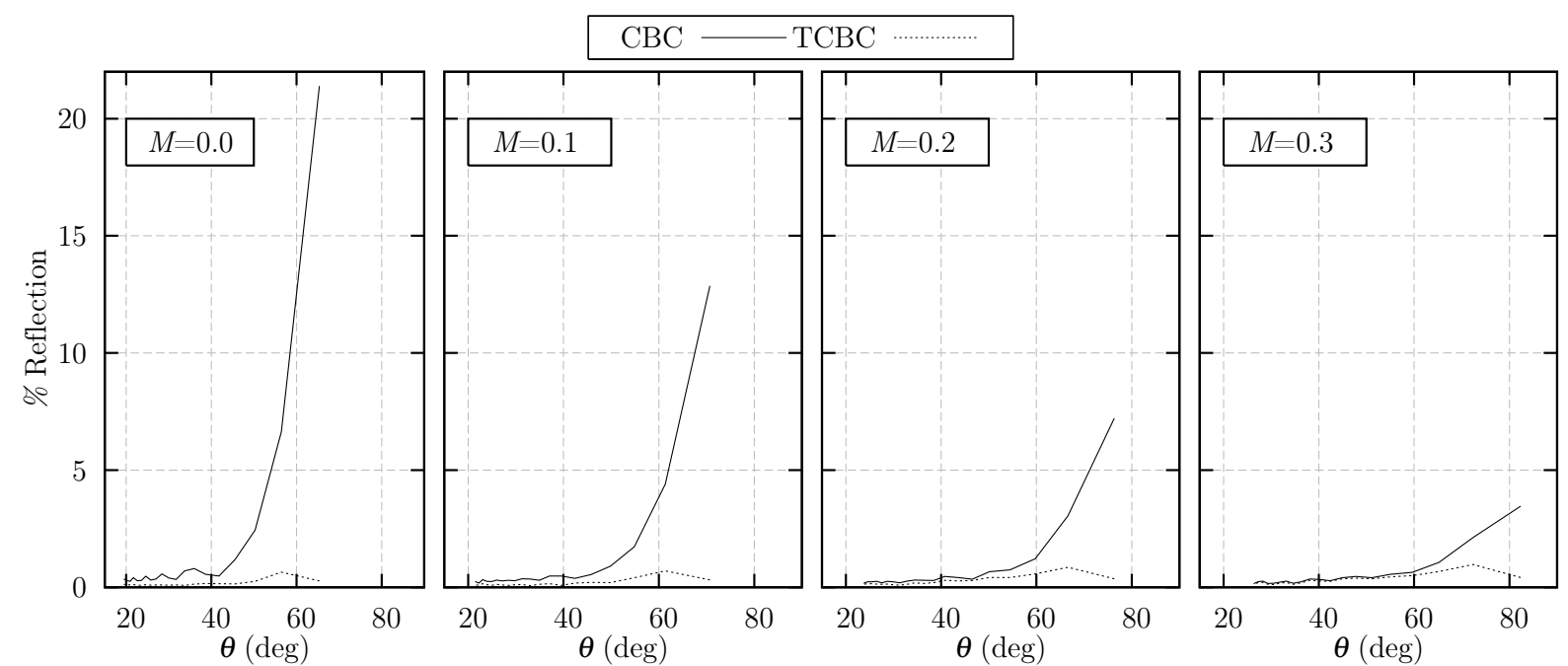

Figure 13. Comparison of numerical reflections from the zonal TCBC and CBC methods for varied wave angle and Mach number.

Additional comparison between the zonal TCBC and CBC methods is given in Figure 14, which compares contours of numerical reflection for various wave angles and ratios of $\lambda_{x} / L$ at $M=0$. While the zonal CBC method shows a dependence on both $\theta$ and $\lambda_{x} / L$, the zonal TCBC method appears to be insensitive to these parameters. Numerical reflections of less then $3 \%$ are seen at all tested conditions.

Figures 13 and 14 have shown that the zonal TCBC method gives good non-reflective performance that appears to be insensitive to Mach number, wave angle, and the relative size of the wave in comparison to the damping zone. Therefore, this method provides a generic non-reflective condition that does not require modification between cases. Although tunable parameters do exist in the method, such as $A, B$, and $\beta$, the values chosen in the current work do not need to be modified in order to give good performance in a variety of cases.

The zonal TCBC method is currently applicable only to the LEE. However, extensions to obtain similar performance with other governing equations may be possible. 

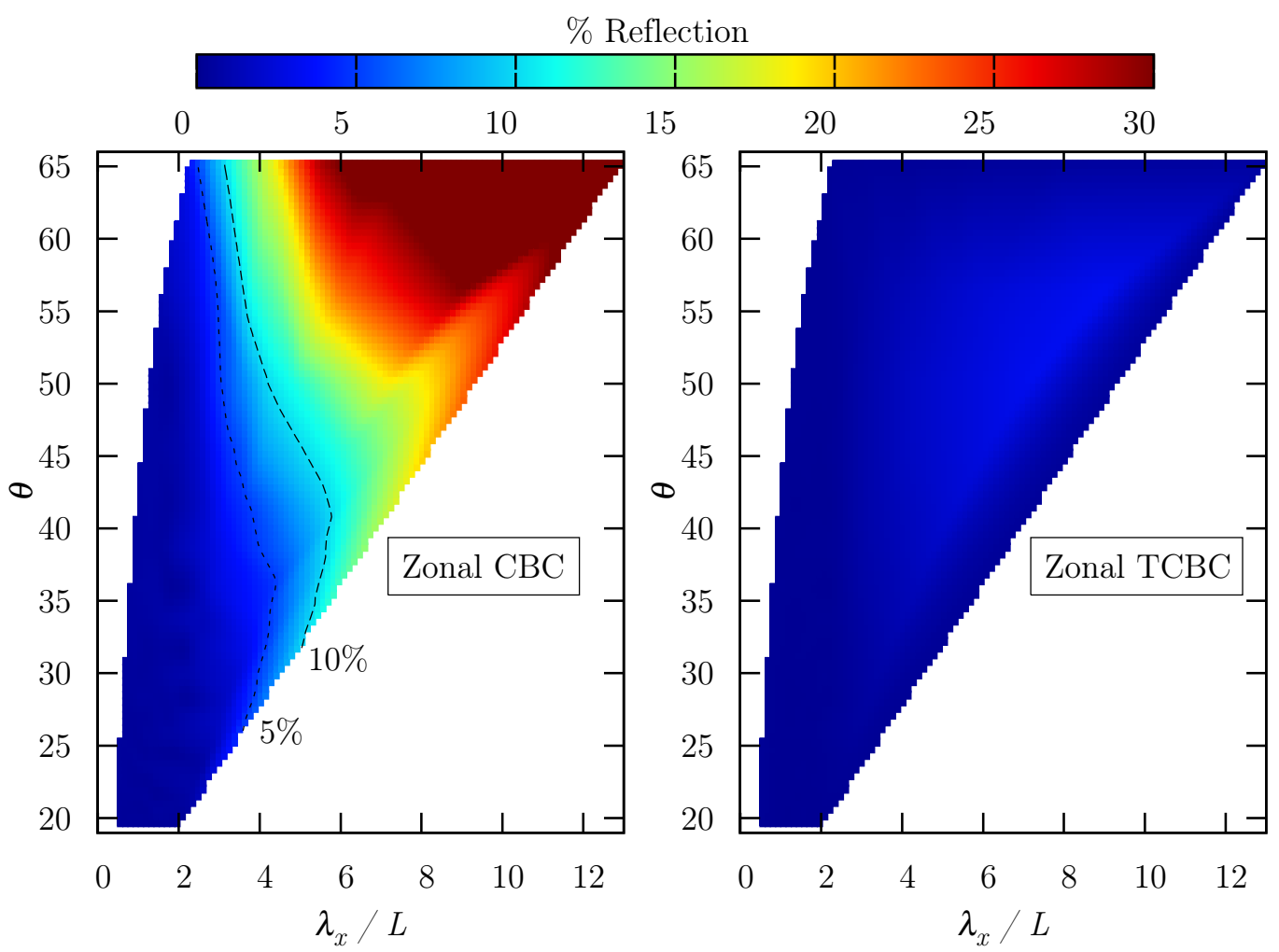

Figure 14. Contours of numerical reflection with varying $\lambda_{x} / L$ and $\theta$, for the Buffer Zone and zonal CBC methods at $M=0$.

\section{Application to Airfoil Turbulence-Interaction Noise Simulation}

This section applies the zonal TCBC boundary condition to the simulation of airfoil turbulence interaction noise. This is done to demonstrate the performance on a realistic problem.

The simulation considers the noise radiated from a NACA 0012 airfoil in $M=0.5$ flow encountering synthesised turbulence. Simulations of this type have been performed by previous authors such as Gill et al. ${ }^{29,33}$ and Kim et al., ${ }^{26}$ among others. The methodology of this case is now outlined. However, additional detail is given in Gea-Aguilera et al., ${ }^{34}$ which uses the same methodology. The purpose of Gea-Aguilera et al. ${ }^{34}$ is to study turbulence interaction noise, whereas the current work is using this case only to demonstrate the performance of the proposed non-reflective boundary condition.

The simulation considers an isolated NACA 0012

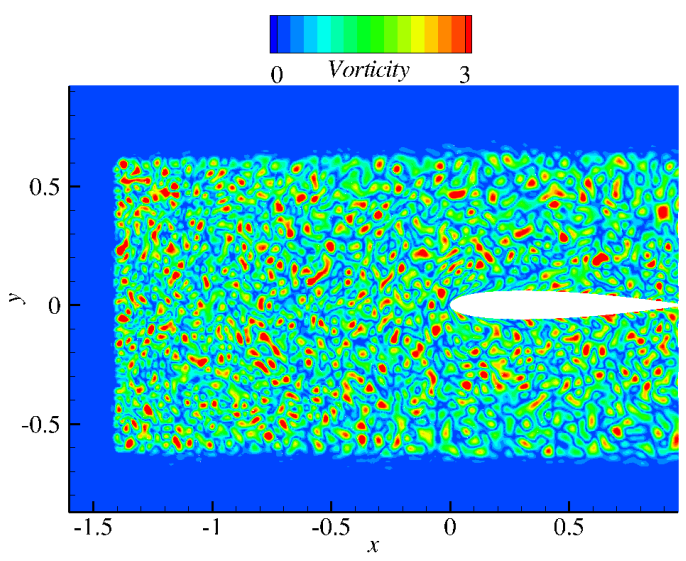

Figure 15. Contours of non-dimensional vorticity. airfoil, with chord of $1 \mathrm{~m}$, in the centre of a domain. Synthesised turbulence is injected into the domain upstream of the airfoil, and is propagated towards the airfoil by the meanflow as frozen turbulence. The meanflow is inviscid and non-uniform. The boundary condition on all domain edges is the proposed zonal TCBC condition. This requires the use of both an inflow and outflow characteristic formulation. However, this paper is concerned with outflow boundary conditions. Therefore, details of the inflow zonal TCBC condition are not included for brevity. The various parameters $\alpha, \beta, A$, and $B$, are set as described in previous sections of this paper, and the TCBC is applied over a zone of 20 grid points from the edge of the 
domain. A Ffowcs-Williams and Hawkings solver is used to make noise predictions in the far-field.

The turbulence is injected from 2 chordlengths upstream of the airfoil. The turbulence synthesis method has been developed by Gea-Aguilera et al., ${ }^{34}$ and is described in the companion paper. This turbulence synthesis method allows the injection of isotropic turbulence in a localised region, as shown in Figure 15. The injected turbulence follows the Von-Kármán spectrum, with turbulence properties defined as $\Lambda=0.07$ $\mathrm{m}$ and $\sqrt{\overline{w^{2}}}=0.04 U_{x}$, where $\Lambda$ is the turbulence integral lengthscale and $\sqrt{\overline{w^{2}}}$ is the turbulence intensity.

Previous authors, such as Gill et al. ${ }^{29}$ and Kim et al. ${ }^{26}$ have used domains which extend to approximately 8 chord lengths from the airfoil in all directions. Kim et al. ${ }^{26}$ solved the Euler equations, and therefore required a domain that is sufficiently large to provide a correct potential flow solution. Gill et al. ${ }^{29}$ solved the LEE equations which can use smaller domains. However, a large domain was required to prevent numerical reflections. Gill et al. used an explicit buffer zone method, combined with some grid stretching in order to remove reflections.

In the current study, four simulations are conducted with varying domain size. The intention is to demonstrate that the zonal TCBC method performs sufficiently well in preventing reflections, such that the domain size can be significantly reduced without affecting accuracy. This will sigificantly reduce the number of cells in the simulation, and thus reduce the computational expense. Domains are used that extend to $8,6,4$, and 3 chordlengths away from the airfoil in all directions. The grid density is uniform in all cases, corresponding to at least 12 points per vortical wavelength in all directions. Therefore, the effects of grid stretching are not considered in the current study.

Figure 16 shows instantaneous contours of the pressure $p$ (non-dimensionalized by $\rho c_{0}^{2}$ ) that is radiated from the airfoil due to the turbulence interaction mechanism. In each case, the entire simulated domain is shown. Figure 16 qualitatively shows that the acoustic pressure waves do not appear to be affected by the edges of the domain, and are not affected by the changes in domain size between the four simulations. An exception to this is at the domain edge directly downstream of the airfoil. In this region, unphysical pressure disturbances can be seen that correspond to the vortical turbulence encountering the zonal TCBC region. However, because these pressure disturbances appear inside the zonal TCBC region, they are contained and do not radiate back into the domain. This effect was also shown by Sandberg and Sandham ${ }^{12}$ Therefore, Figure 16 qualitatively demonstrates that the zonal TCBC method can provide good performance in the presence of both acoustic and vortical waves.

Far-field noise predictions were made for an observer at $90^{\circ}$ to the freestream direction and at a radius of $100 \mathrm{~m}$. Figure 17 compares the noise predictions for varying domain size with an analytical prediction of the noise from a flat plate, as given by Amiet's theory. ${ }^{35}$ For $f<1000 \mathrm{~Hz}$ a good agreement is seen between all methods. Above $f=1000 \mathrm{~Hz}$ the numerical predictions deviate from the analytical prediction due to the thickness of the NACA 0012 airfoil. This effect is discussed in Gea-Aguilera et al. ${ }^{34}$ The noise predictions from the various domain sizes are in agreement to within $2 \mathrm{~dB}$ at all frequencies. This suggests that the noise predictions are unaffected by the changes in domain size, and that a domain which extends to 3 airfoil chords in all directions is sufficient if the zonal TCBC non-reflective boundary condition is used. 

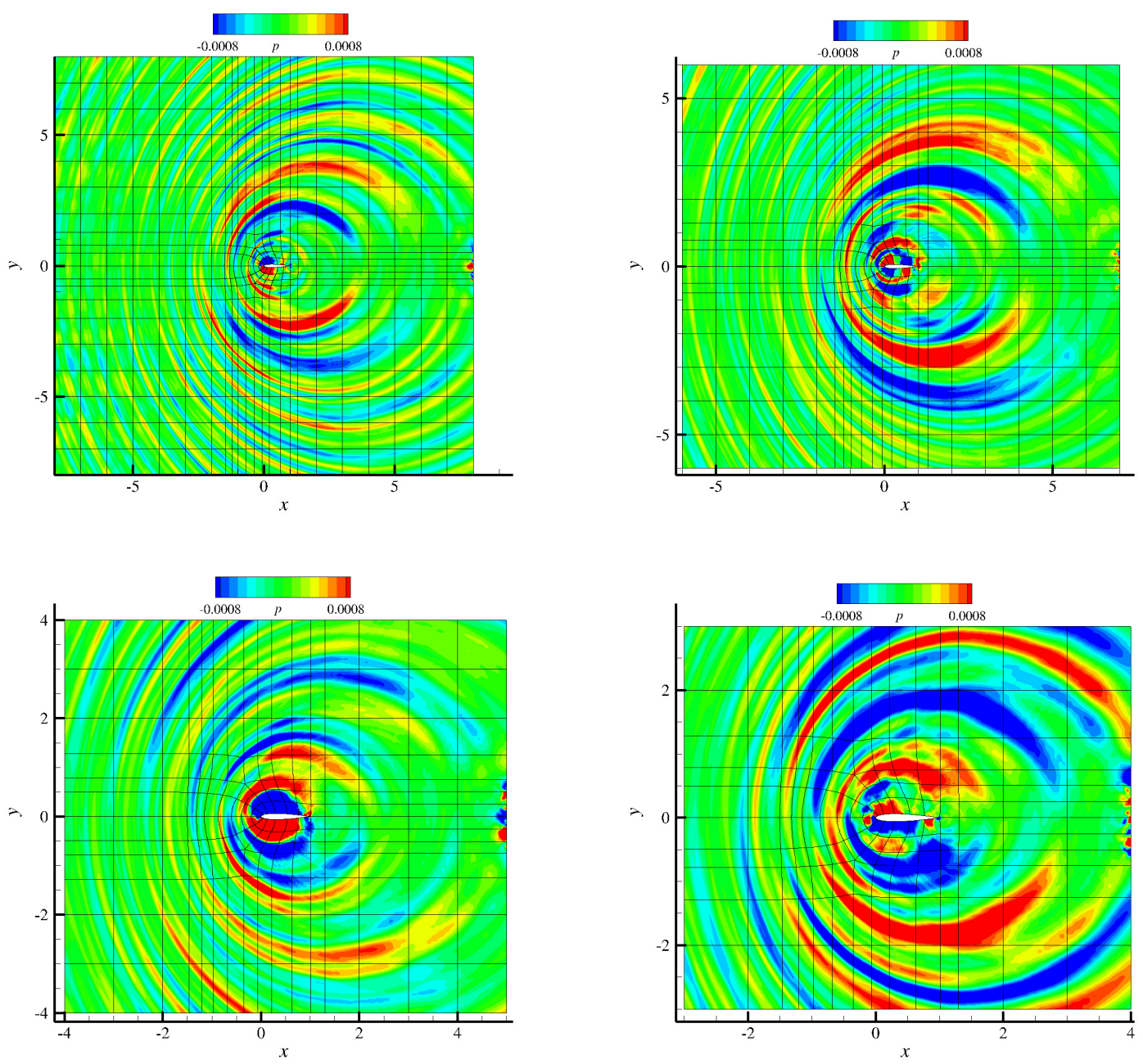

Figure 16. Contours of instantaneous $p$ for simulations using varying domain sizes.

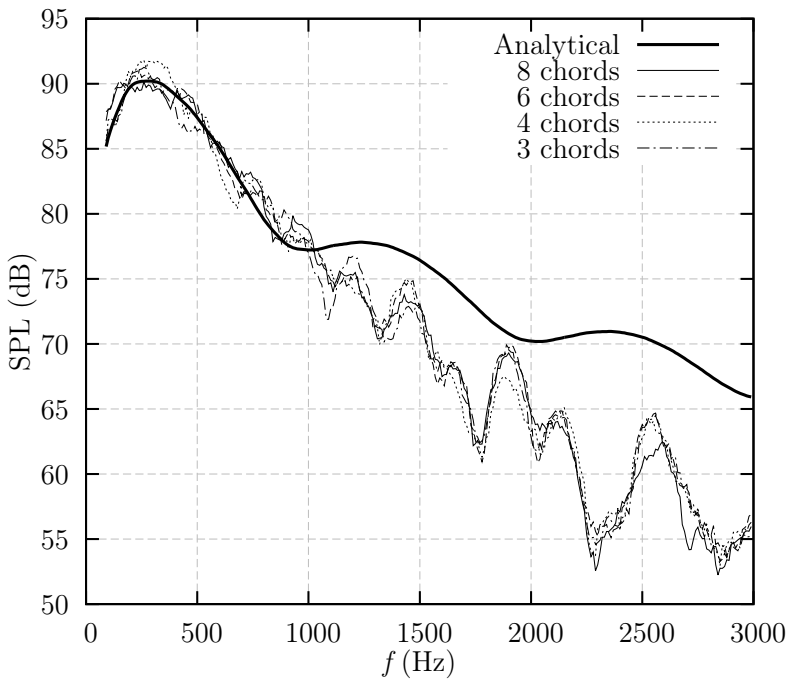

Figure 17. Comparison of noise spectra using varying domain size with noise predictions from Amiet's ${ }^{35}$ analytical theory. 


\section{Application to an Engine Duct Mode Simulation}

The TCBC boundary condition was also applied to a three-dimensional duct mode simulation in order to demonstrate the non-reflective performance. The propagation of a single spinning mode through a generic engine bypass duct was simulated. A circumferential mode number of $m=12$ was used, and the frequency of the disturbance was $1500 \mathrm{~Hz}$. This configuration was previously studied by Chen et al. ${ }^{36}$ The disturbance was injected at the domain inflow and allowed to propagate over a non-uniform meanflow solution that was obtained with a RANS calculation using a commercial steady flow solver. To reduce the computational cost, a 1/12th segment of the engine was simulated and periodic conditions were applied. The simulation result was azimuthally copied in a post-processing step to provide the full engine image that is shown in Figure 18. In order to prevent simulation instabilities related to the non-uniformity of the meanflow, a Gradient Term Suppression (GTS) technique was used, where the governing equations are solved by ignoring spatial gradient terms across shear layers. ${ }^{37}$ The TCBC boundary condition was used at all outflow domain boundaries, in a zonal region that extended to 15 points from the domain edge.

For brevity, detailed analysis is not made on the bypass duct case in this paper, as the purpose is to qualitatively demonstate the ability of the TCBC condition in preventing acoustic reflections in threedimensional simulations. The simulation time allowed 50 periods of the duct mode to propagate from the domain inflow.

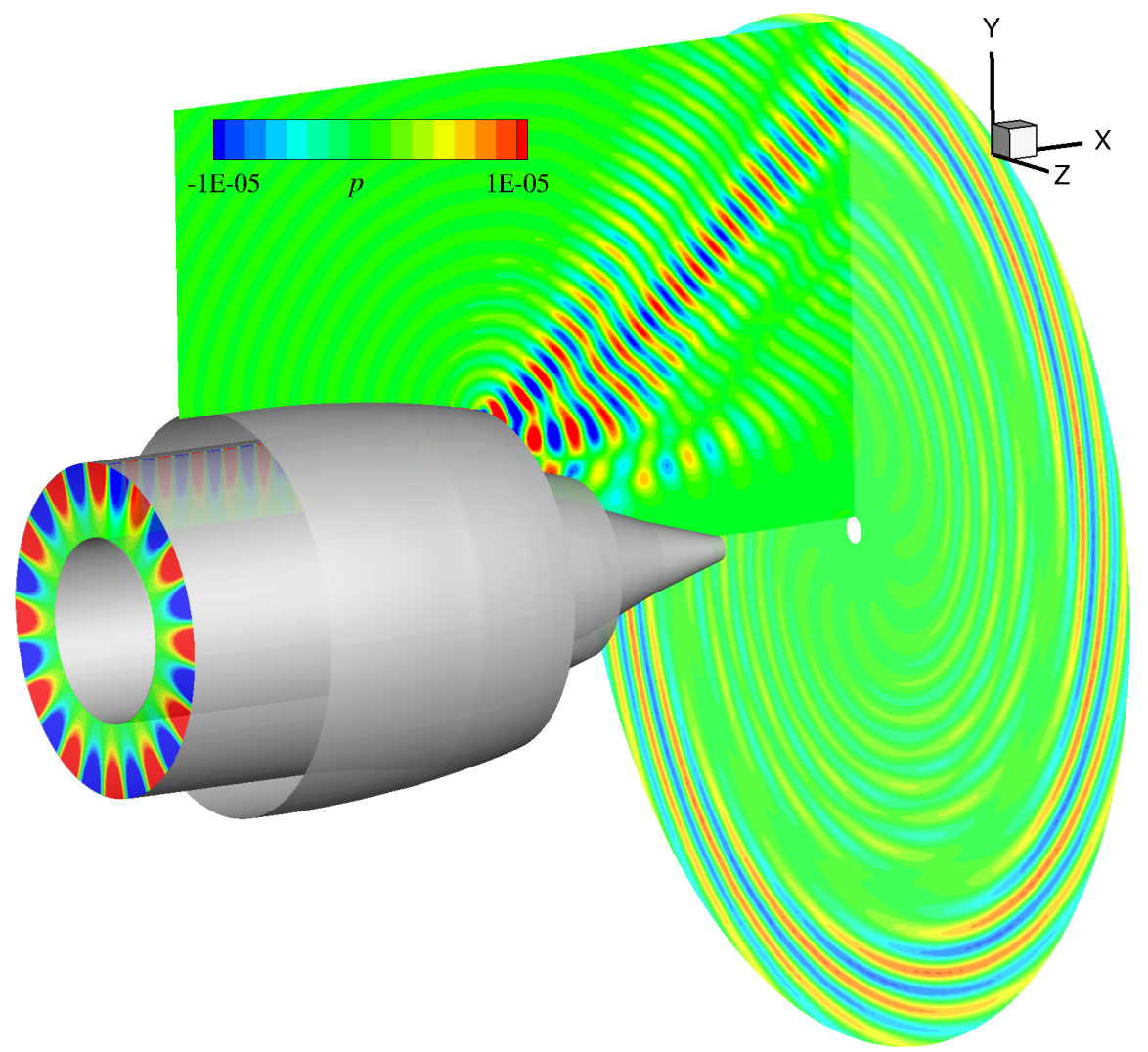

Figure 18. Contours of non-dimensional instantaneous acoustic pressure for a single mode radiating from a generic bypass engine duct configuration.

Figure 18 shows contours of instantaneous non-dimensional acoustic pressure in the $x-y$ and $y-z$ planes. The full extent of the domain is shown in these planes. The propagating acoustic waves are seen to cleanly pass through the domain boundary and do not give a discernable acoustic reflection. This result further confirms the ability of the TCBC boundary condition to prevent acoustic reflections, and demonstrates the effectiveness in three-dimensional simulations. 


\section{Summary}

Effective non-reflective boundary conditions are a key criteria for successful CAA simulations. They are required in order to prevent spurious reflected waves from simulation boundaries interfering with acoustic results. Previous authors have suggested a variety of methods to provide a non-reflective boundary condition, using methods based on characteristics, far-field approximations, and buffer zones, for example.

This paper has used the inexpensive plane wave test configuration of Richards et al. ${ }^{1}$ to quantitatively measure the performance of different non-reflective boundary conditions. The performance of buffer zone methods, far-field approximations, and characteristic boundary conditions (CBCs) has been assessed with varying Mach number, wave angle, and frequency. Where tunable parameters have been required in the various methods, parameter studies have been made to optimize the value of these parameters in order to provide good comparison. A new $\mathrm{CBC}$ is proposed and tested in addition to testing previous methods. The new zonal transverse characteristic boundary condition (TCBC) improves Thompson's CBC by adding an estimate for the tranverse characteristic terms (in a similar but modified manner to the method of Yoo and $\operatorname{Im}^{8}$ ), and by forcing the boundary condition over a ramped zone as suggested by Sandberg and Sandham. ${ }^{12}$

In general, the performance of the tested non-reflective boundary conditions is best for waves that are normal to the edge of the domain, and for wavelengths that are small in comparison to the length of the damping zone that is used. The best non-reflective performance was found from the far-field approximation method of Tam and Webb, ${ }^{14}$ which did not give a discernible reflection in any configuration that was tested. However, this approach is not generic as it requires the position of the noise source to be known in relation to the domain boundary. Buffer zone approaches can perform well for waves that are normal to the boundary, but the performance is degraded for acute wave angles. The local CBC of Thompson ${ }^{4,5}$ gave the least effective performance of all the tested methods. However, the proposed zonal TCBC method provides the best generic performance across all tested combinations of Mach number, wave angle and wave frequency.

The performance of the zonal TCBC method has been demonstrated on a two-dimensional airfoil turbulenceinteraction noise case, which includes outgoing acoustic and vortical waves, and also on a three-dimensional duct mode case.

The parameters $A$ and $B$ in the TCBC method have been obtained empirically in the current work. Future work is recommended to improve understanding of why these parameter values give optimal nonreflective performance. Additionally, the current study is limited to simulations that solve the linearized Euler equations. Future studies to consider simulations solving the Euler and Navier-Stokes equations would be beneficial.

\section{References}

\footnotetext{
${ }^{1}$ Richards, S., ZhangR, X., Chen, X., and Nelson, P. A., "The evaluation of non-reflecting boundary conditions for duct acoustic computation," Journal of Sound and Vibration, Vol. 270, No. 3, Feb. 2004, pp. 539-557.

${ }^{2}$ Colonius, T., "Modelling Artificial Boundary Conditions for Compressible Flow," Annual Review of Fluid Mechanics, Vol. 36, No. 1, Jan. 2004, pp. 310-345.

${ }^{3}$ Tam, C., "Advances in Numerical Boundary Conditions for Computational Aeroacoustics," AIAA Journal, , No. 97-1774, 1997.

${ }^{4}$ Thompson, K. W., "Time Dependent Boundary Conditions for Hyperbolic Systems, I," Journal of Computational Physics, Vol. 68, 1987, pp. 1-24.

${ }^{5}$ Thompson, K. W., "Time Dependent Boundary Conditions for Hyperbolic Systems, II," Journal of Computational Physics, Vol. 89, 1990, pp. 439-461.

${ }^{6}$ Poinsot, T. and Lele, S. K., "Boundary Conditions for Direct Simulations of Compressible Viscous Flows," Journal of Computational Physics, Vol. 101, 1992, pp. 104-129.

${ }^{7}$ Giles, M., "Nonreflecting Boundary Conditions for Euler Equation Calculations," AIAA Journal, Vol. 28, No. 28, 1990, pp. 2050-2058.

${ }^{8}$ Yoo, C. S. and Im, H. G., "Characteristic Boundary Conditions for Simulations of Compressible Reacting Flows with Multi-Dimensional, Viscous and Reaction Effects," Combustion Theory and Modelling, Vol. 11, No. 2, 2007, pp. 259-286.

${ }^{9} \mathrm{Liu}$, Q. and Vasilyev, O. V., "Nonreflecting Boundary Conditions Based on Nonlinear Multidimensional Characteristics," International Journal for Numerical Methods in Fluids, Vol. 62, 2009, pp. 24-55.

${ }^{10}$ Kim, J. W., Lau, A. S. H., and Sandham, N. D., "CAA Boundary Conditions for Airfoil Noise Due to High-Frequency Gusts," Periodica Engineering, Vol. 6, No. 6, Jan. 2010, pp. 244-253.

${ }^{11}$ Collis, S. S. and Lele, S. K., "A Computational Approach to Swept Leading-Edge Receptivity," AIAA Journal, , No. 1996-0180, 1996.

${ }^{12}$ Sandberg, R. D. and Sandham, N. D., "Nonreflecting Zonal characteristic Boundary Condition for Direct Numerical Simulation of Aerodynamic Sound," AIAA Journal, Vol. 44, No. 2, 2006, pp. 402-405.
} 
${ }^{13}$ Bayliss, A. and Turkel, E., "Radiation Boundary Conditions for Wave-Like Equations," Communications on Pure and Applied Mathematics, Vol. 33, 1980, pp. 708-725.

${ }^{14}$ Tam, C. and Webb, J., "Dispersion-Relation-Preserving Finite Difference Schemes for Computational Acoustics," Journal of Computational Physics, Vol. 107, 1993, pp. 262-281.

${ }^{15}$ Tam, C. and Dong, Z., "Radiation and Outflow Boundary Conditions for Direct Computation of Acoustic and Flow Disturbances in a Nonuniform Mean Flow," Journal of Computational Acoustics, Vol. 4, No. 2, 1996, pp. 175-201.

${ }^{16}$ Wasistho, B., Geurts, B. J., and Kuerten, J. G. M., "Simulation Technique for Spatially Evolving Instabilities in Compressible Flow Over a Flat Plate," Computers and Fluids, Vol. 26, No. 7, 1997, pp. 713-739.

${ }^{17}$ Colonius, T., Lele, S. K., and Moin, P., "Boundary Conditions for Direct Computation of Aerodynamic Sound Generation," AIAA Journal, Vol. 31, No. 9, 1993, pp. 1574-1582.

${ }^{18}$ Freund, J. B., "Proposed Inflow/Outflow Boundary Condition for Direct Computation of Aerodynamic Sound," AIAA Journal, Vol. 35, No. 4, 1997, pp. 740-742.

${ }^{19}$ Berenger, J. P., "A Perfectly Matched Layer for the Absoption of Electromagnetic Waves," Journal of Computational Physics, Vol. 114, 1994, pp. 182-200.

${ }^{20} \mathrm{Hu}, \mathrm{F}$., Hussaini, M. Y., and Manthey, J., "Low-Dissipation and Low-Dispersion Runge-Kutta Schemes for Computational Aeroacoustics," Journal of Computational Physics, Vol. 124, No. 52, 1996, pp. 177-191.

${ }^{21}$ Hixon, R., Shih, S., and Mankbadi, R. R., "Evaluation of Boundary Conditions for Computational Aeroacoustics," Tech. Rep. AIAA-95-0160, NASA, Cleveland, 1995.

${ }^{22}$ Bogey, C. and Bailly, C., "Three-Dimensional Non-Reflective Boundary Conditions for Acoustic Simulations: Far Field Formulation and Validation Test Cases," Acta Acustica United, Vol. 88, 2002, pp. 463-471.

${ }^{23} \mathrm{Hu}$, F., "A Stable, Perfectly Matched Layer for Linearized Euler Equations in Unsplit Physical Variables," Journal of Computational Physics, Vol. 173, 2001, pp. 455-480.

${ }^{24}$ Fosso, A., Deniau, H., Lamarque, N., and Poinsot, T., "Comparison of Outflow Boundary Conditions for Subsonic Aeroacoustic Simulations," International Journal for Numerical Methods in Fluids, Vol. 68, No. 10, 2012, pp. 1207-1233.

${ }^{25}$ Wilson, A. G., "A Method for Deriving Tone Noise Information From CFD Calculations on the Aeroengine Fan Stage," NATO RTO-AVT Symposium on Developments in Computational Aero- and Hydro-Acoustics, Manchester UK, 2001.

${ }^{26}$ Kim, J. W., Lau, A. S. H., and Sandham, N. D., "Proposed Boundary Conditions for Gust-Airfoil Interaction Noise," AIAA Journal, Vol. 48, No. 11, Nov. 2010, pp. 2705-2710.

${ }^{27}$ Kim, J. W. and Lee, D. J., "Generalized Characteristic Boundary Conditions for Computational Aeroacoustics, Part 2," AIAA Journal, Vol. 42, No. 1, 2004, pp. 47-55.

${ }^{28}$ Zhang, X., Chen, X., and Nelson, P. A., "Computation of Spinning Modal Radiation from an Unflanged Duct," AIAA Journal, Vol. 42, No. 6, 2004, pp. 1795-1801.

${ }^{29}$ Gill, J., Zhang, X., and Joseph, P. F., "Symmetric airfoil geometry effects on leading edge noise." The Journal of the Acoustical Society of America, Vol. 134, No. 4, Oct. 2013, pp. 2669-80.

${ }^{30}$ Zhang, X. and Chen, X., "Linearised Divergence Equations for Sound Propagation," 17th AIAA/CEAS Aeroacoustics Conference, Portland, Oregon, No. 2011-2930, 2011.

${ }^{31}$ Liu, W., Kim, J. W., Zhang, X., Angland, D., and Caruelle, B., "Landing Gear Noise Prediction using High-Order Finite Difference Schemes," Journal of Sound and Vibration, Vol. 332, No. 14, 2013, pp. 3417-3534.

${ }^{32}$ Hixon, R., "Prefactored Small-Stencil Compact Schemes," Journal of Computational Physics, Vol. 165, No. 2, Dec. 2000, pp. $522-541$.

${ }^{33}$ Gill, J., Zhang, X., Joseph, P. F., and Node-Langlois, T., "Reduced Dimension Modeling of Leading Edge Turbulent Interaction Noise," 20th AIAA/CEAS Aeroacoustics Conference, Atlanta Georgia, No. 2014-2321, 2014.

${ }^{34}$ Gea-Aguilera, F., Zhang, X., Chen, X., and Gill, J., "Synthetic Turbulence Methods for Leading Edge Noise Predictions," 21st AIAA/CEAS Aeroacoustics Conference, Dallas, 2015.

${ }^{35}$ Amiet, R. K., "Acoustic Radiation from an Airfoil in a Turbulent Stream," Journal of Sound and Vibration, Vol. 41, No. 4, 1975, pp. 407-420.

${ }^{36}$ Chen, X., Huang, X., and Zhang, X., "Sound Radiation from a Generic Bypass Duct with Bifurcations," AIAA Journal, Vol. 47, No. 2, 2008, pp. 429-436.

${ }^{37}$ Zhang, X., Chen, X., and Gill, J., "Gradient Term Filtering for Stable Sound Propagation with Linearized Euler Equations," 20th AIAA/CEAS Aeroacoustics Conference, Atlanta Georgia, No. 2014-3306, 2014. 